\title{
Kinematical distributions of coherent neutrino trident production in gauged $L_{\mu}-L_{\tau}$ model
}

\author{
Takashi Shimomura, ${ }^{1, *}$ and Yuichi Uesaka $\odot^{2, \dagger}$ \\ ${ }^{1}$ Faculty of Education, University of Miyazaki, 1-1 Gakuen-Kibanadai-Nishi, Miyazaki 889-2192, Japan \\ ${ }^{2}$ Faculty of Science and Engineering, Kyushu Sangyo University, \\ 2-3-1 Matsukadai, Higashi-ku, Fukuoka 813-8503, Japan
}

(Received 5 October 2020; accepted 1 February 2021; published 23 February 2021)

\begin{abstract}
We analyze the distributions of energy, the opening angle, and the invariant mass in muonic neutrino trident production processes, $\nu_{\mu} \rightarrow \nu_{\mu} \mu \bar{\mu}$, in a minimal gauged $U(1)_{L_{\mu}-L_{\tau}}$ model, in which the discrepancy of the anomalous magnetic moment of muon can be solved. It is known that the total cross section of neutrino trident production has parameter degeneracy in the new gauge boson mass and gauge coupling constant, and therefore other observables are needed to determine these parameters. From numerical analyses, we find that the muon energy and invariant mass distributions show the differences among the new physics parameter sets with which the total cross section has the same value, while the antimuon energy and opening angle distributions are not sensitive to the parameters.
\end{abstract}

DOI: 10.1103/PhysRevD.103.035022

\section{INTRODUCTION}

The anomalous magnetic moment of muon is a longstanding discrepancy between experimental measurements [1,2] and theoretical predictions [3-6]. The recent result of the Standard Model (SM) prediction [4] shows that the difference of the anomalous magnetic moment, $a_{\mu} \equiv\left(g_{\mu}-2\right) / 2$, from the measurements reaches to [6]

$$
\Delta a_{\mu} \equiv a_{\mu}^{\mathrm{Exp}}-a_{\mu}^{\mathrm{SM}}=(27.06 \pm 7.26) \times 10^{-10} .
$$

Thus the SM predictions are $3.7 \sigma$ lower than the experimental measurements. Extensive studies on the theoretical side have been made, however the discrepancy cannot be resolved within the SM of particle physics (for review, see [7] for an example). The E989 experiments at Fermilab [8] and the E34 experiment at J-PARC [9] are ongoing and will reduce experimental uncertainties by a factor of four, which could confirm the discrepancy at the $5 \sigma$ level. Once the discrepancy is confirmed, it will be a clear signature of new physics (NP) beyond the SM.

Many new physics models have been proposed to explain the discrepancy of $a_{\mu}$ by extending the SM. One of the simplest extensions in this regard is to impose an

\footnotetext{
uesaka@ip.kyusan-u.ac.jp

†shimomura@cc.miyazaki-u.ac.jp
}

Published by the American Physical Society under the terms of the Creative Commons Attribution 4.0 International license. Further distribution of this work must maintain attribution to the author(s) and the published article's title, journal citation, and DOI. Funded by SCOAP . extra $U(1)$ gauge symmetry on the $\mathrm{SM}$, so that the contribution of a new gauge boson accounts for the deviation of the muon anomalous magnetic moment. Among such extensions, the $U(1)$ symmetry gauging muon flavor number minus the tau flavor number, or $L_{\mu}-L_{\tau}$ [10-12], has been gaining attention in recent years. In [13], it was shown that a gauge boson of the $U(1)_{L_{\mu}-L_{\tau}}$ symmetry can explain the deviation without conflicting experimental searches, provided that the mass and gauge coupling are $\mathcal{O}(100) \mathrm{MeV}$ and $\mathcal{O}\left(10^{-4}\right)$, respectively. Possibilities of searches for this light and weakly interacting gauge boson have been studied in [14-22]. Other studies based on the $L_{\mu}-L_{\tau}$ symmetry have also been done, such as the cosmic neutrino spectrum observed at IceCube $[23,24]$, neutrino mass and mixing [25-28], dark matter [29-31], the baryon asymmetry of the Universe [32], and meson decay [33-35], for recent works. Light gauge bosons interacting with muonic leptons can contribute to neutrino trident production (NTP) processes such as $\nu_{\mu}+N \rightarrow \nu_{\mu}+\mu+\bar{\mu}+N$ [36-43]. It was shown in [13,44] that the NTP processes can set severe bounds on the gauge boson mass and the gauge coupling. Utilizing the results of the CHARM-II [45], CCFR [46], and NuTeV [47] experiments, one finds that the region of the mass above $\mathcal{O}(100) \mathrm{MeV}$ and the gauge coupling above $\mathcal{O}\left(10^{-3}\right)$ are excluded. The analyses of the NTP processes in the SM or new physics models have also been done for future planned experiments, DUNE [48-53], SHiP [48,49], MINOS, NO $\nu$ A, MINERvA [51], and MicroBooNE [54], and ongoing experiments, T2K $[15,51]$ and IceCube [55-57], taking into account coherent and diffractive processes. In particular, the liquid argon detector at the near site in the DUNE experiment is 
expected to observe $\mathcal{O}(100)$ events of muonic NTP processes [51-53]. As presented in [13,52,53], the contours of the total cross section of the NTP processes are obtained as a function of new physics parameters, i.e., the mass and coupling constant of the new gauge bosons. This results in the fact that the new physics parameters cannot be uniquely determined by using the single value of the total cross section. In other words, the total cross section has parameter degeneracy in the new physics parameters. To determine or further constrain the new physics parameters, one needs other observables in addition to the total cross section. One of these observables will be the differential cross sections that are generally measured simultaneously in experiments. When the differential cross sections show the differences of the new physics parameters for the fixed values of the total cross section, we can determine or constrain the parameters by combining the information from the differential and total cross sections. As a first step for this purpose, we analyze the parameter dependences of the differential cross sections with respect to the energies, the opening angles, and the invariant masses of the final state muons in a minimal $L_{\mu}-L_{\tau}$ model. Our results will show which distributions should be used for detailed analyses for the determination of the parameters.

This paper is organized as follows: In Sec. II, we briefly review a minimal gauged $L_{\mu}-L_{\tau}$ model and present relevant interactions. The amplitudes and cross section of NTP processes are given in Sec. III. Then, we show our numerical results for the distributions with respect to the energy, the opening angle, and the invariant mass of the muon pair in Sec. IV. Section V is devoted to summary.

\section{MINIMAL $L_{\mu}-L_{\tau}$ MODEL}

We start our discussion by reviewing a minimal gauged $L_{\mu}-L_{\tau}$ model. The gauge sector of the SM is extended by adding the $U(1)_{L_{\mu}-L_{\tau}}$ gauge symmetry under which mu and tau flavored leptons among the SM fermions are charged. The charge assignment for leptons under this symmetry is shown in Table I. In the table, $e, \mu$, and $\tau$ represent charged leptons, and $\nu_{e}, \nu_{\mu}$, and $\nu_{\tau}$ are corresponding left-handed neutrinos, respectively. Up-type and down-type quarks as well as the Higgs boson are singlets under the $U(1)_{L_{\mu}-L_{\tau}}$ gauge symmetry.

The relevant interaction Lagrangian for the NTP processes is given by

TABLE I. The charge assignment of the gauged $U(1)_{L_{\mu}-L_{\tau}}$ model.

\begin{tabular}{lllcccr}
\hline \hline & $e$ & $\mu$ & $\tau$ & $\nu_{e}$ & $\nu_{\mu}$ & $\nu_{\tau}$ \\
\hline$U(1)_{L_{\mu}-L_{\tau}}$ & 0 & 1 & -1 & 0 & 1 & -1 \\
\hline \hline
\end{tabular}

$$
\begin{aligned}
\mathcal{L}_{\text {int }}= & e A_{\rho} J_{\mathrm{em}}^{\rho}-\frac{4 G_{F}}{\sqrt{2}}\left[\bar{\nu}_{\ell_{4}} \gamma_{\rho} \nu_{\ell_{3}}\right]\left[\bar{\ell}_{2} \gamma^{\rho}\left(g_{L} P_{L}+g_{R} P_{R}\right) \ell_{1}\right] \\
& +g^{\prime} Z_{\rho}^{\prime} J_{Z^{\prime}}^{\rho}
\end{aligned}
$$

where $e, A^{\rho}$, and $J_{\mathrm{em}}^{\rho}$ are the elementary electric charge, photon field, and electromagnetic current of the SM, respectively. In the second term of Eq. (2), $G_{F}$ is the Fermi coupling constant, and $\ell_{1,2}$ represent charged leptons $(e, \mu, \tau)$ and $\nu_{\ell_{3,4}}$ represent neutrinos $\left(\nu_{e}, \nu_{\mu}, \nu_{\tau}\right)$. The lefthanded (right-handed) projection operator is denoted as $P_{L(R)}$. The constants $g_{L}$ and $g_{R}$ are given by

$$
\begin{aligned}
& g_{L}=\left(-\frac{1}{2}+\sin ^{2} \theta_{W}\right) \delta_{\ell_{1}, \ell_{2}} \delta_{\ell_{3}, \ell_{4}}+\delta_{\ell_{1}, \ell_{4}} \delta_{\ell_{2}, \ell_{3}}, \\
& g_{R}=\sin ^{2} \theta_{W} \delta_{\ell_{1}, \ell_{2}} \delta_{\ell_{3}, \ell_{4}},
\end{aligned}
$$

where $\theta_{W}$ is the Weinberg angle and each $\ell_{i}(i=1-4)$ represents the flavor of the charged leptons or neutrinos. From Eq. (3), $g_{L}$ for muonic $\left(\nu_{\mu} \rightarrow \nu_{\mu} \mu \bar{\mu}\right)$ and tauonic $\left(\nu_{\mu} \rightarrow \nu_{\mu} \tau \bar{\tau}\right)$ NTP processes is

$$
g_{L}=\left\{\begin{array}{cc}
\frac{1}{2}+\sin ^{2} \theta_{W} & \left(\nu_{\mu} \rightarrow \nu_{\mu} \mu \bar{\mu}\right), \\
-\frac{1}{2}+\sin ^{2} \theta_{W} & \left(\nu_{\mu} \rightarrow \nu_{\mu} \tau \bar{\tau}\right),
\end{array}\right.
$$

respectively, while from Eq. (3b), $g_{R}$ is $\sin ^{2} \theta_{W}$ for both processes. The third term of Eq. (2) is the interaction of the $L_{\mu}-L_{\tau}$ gauge boson $Z^{\prime}$ with the gauge coupling constant $g^{\prime}$. The $L_{\mu}-L_{\tau}$ gauge current, $J_{Z^{\prime}}^{\rho}$, is given by

$$
J_{Z^{\prime}}^{\rho}=\bar{\mu} \gamma^{\rho} \mu-\bar{\tau} \gamma^{\rho} \tau+\overline{\nu_{\mu}} \gamma^{\rho} \nu_{\mu}-\overline{\nu_{\tau}} \gamma^{\rho} \nu_{\tau}
$$

In this work, we consider a minimal $L_{\mu}-L_{\tau}$ model in which the gauge kinetic mixing term between the $U(1)_{Y}$ hypercharge and the $U(1)_{L_{\mu}-L_{\tau}}$ symmetries is absent at tree-level, even though the gauge kinetic mixing can be generated radiatively via loop diagrams in which muon, tau, and neutrinos propagate. The loop-induced kinetic mixing parameter between the photon $\gamma$ and $Z^{\prime}$ can be obtained at the one-loop level by evaluating Fig. 1 as

$\epsilon\left(q^{2}\right)=\frac{8 e g^{\prime}}{(4 \pi)^{2}} \int_{0}^{1} d x x(1-x) \log \left(\frac{m_{\tau}^{2}-x(1-x) q^{2}}{m_{\mu}^{2}-x(1-x) q^{2}}\right)$,

where $q$ is the four momentum carried by $\gamma$ and $Z^{\prime}$, and $m_{\mu}$ and $m_{\tau}$ are the masses of muon and tau, respectively. The approximate expression of Eq. (6) is given by 


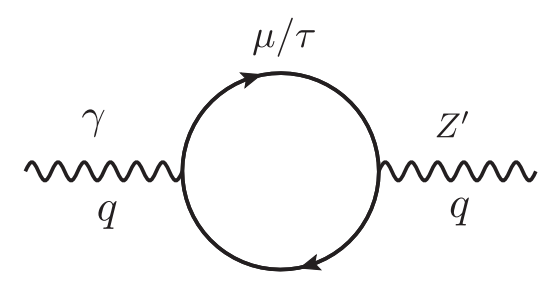

FIG. 1. Loop-induced kinetic mixing between the photon $\gamma$ and the $Z^{\prime}$ boson.

$\epsilon\left(q^{2}\right) \simeq \begin{cases}\frac{8 e g^{\prime}}{3(4 \pi)^{2}} \log \frac{m_{\tau}}{m_{\mu}}, & \left(q^{2} \ll 4 m_{\mu}^{2}\right), \\ -\frac{6 e g^{\prime}}{(4 \pi)^{2}}\left\{\left(\frac{m_{\tau}^{2}}{q^{2}}-\frac{m_{\mu}^{2}}{q^{2}}\right)+i \pi\left(\frac{m_{\tau}^{4}}{q^{4}}-\frac{m_{\mu}^{4}}{q^{4}}\right)\right\}, & \left(q^{2} \gg 4 m_{\tau}^{2}\right) .\end{cases}$

This loop-induced kinetic mixing parameter is about two orders of magnitude smaller than $g^{\prime}$ for $q^{2} \ll 4 m_{\mu}^{2}$. It is further suppressed by a power of $m_{\tau, \mu}^{2} / q^{2}$ for $q^{2} \gg 4 m_{\tau}^{2}$. For the intermediate $q^{2}\left(4 m_{\mu}^{2}<q^{2}<4 m_{\tau}^{2}\right)$, the real and imaginary parts are also two orders of magnitude smaller than $g^{\prime}$. Therefore, it is negligible compared with $g^{\prime}$. We drop the loop-induced kinetic mixing parameter in our analyses. There also exists the loop-induced kinetic mixing between $Z^{\prime}$ and the neutral weak boson $Z$. However, since the energy of the incident neutrinos we consider is smaller than the $Z$ boson mass $m_{Z}$, such a mixing is practically negligible because it is suppressed by $m_{Z}$.

We also assume that the $L_{\mu}-L_{\tau}$ symmetry and the electroweak symmetry are appropriately broken without conflicting with all existing experimental data so that $Z^{\prime}$ can acquire a mass $m_{Z^{\prime}}$ of order $0.01-10 \mathrm{GeV}$. We do not specify the scalar sector of the model and treat $m_{Z^{\prime}}$ as a free parameter in the following analyses. Thus only two parameters, $m_{Z^{\prime}}$ and $g^{\prime}$, are newly introduced to the SM in our setup.

\section{NEUTRINO TRIDENT PRODUCTION PROCESSES}

In this section, the amplitudes and cross sections of the NTP processes in the SM and the minimal gauged

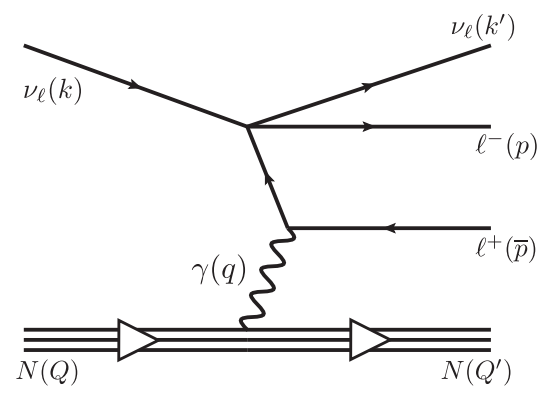

(a)
$U(1)_{L_{\mu}-L_{\tau}}$ model are presented, as well as a brief summary of the experimental results. Depending on the virtuality of the photon $q^{2}$, the appropriate picture of a hadronic target is different. Based on the appropriate hadronic picture, the NTP can be classified by three processes: coherent, diffractive, and deep inelastic, where the incoming neutrino scatters off the nuclei, nucleons, and quarks, respectively. According to Ref. [48], the deep inelastic contribution accounts for at most $1 \%$ of the total NTP cross section, and therefore we do not consider this contribution. For relevant energies of the initial neutrino, the coherent and diffractive processes give comparable contributions. As the first step, we focus on the coherent process in this work.

In the following subsections, the four momenta of the incident neutrino $\left(\nu_{\ell}\right)$ and nucleus $(N)$ are assigned to $k$ and $Q$ while those of outgoing ones are assigned to $k^{\prime}$ and $Q^{\prime}$, respectively. For the lepton $\left(\ell^{-}\right)$and antilepton $\left(\ell^{+}\right)$, the four momenta are assigned to $p$ and $\bar{p}$, and for the virtual photon, the momentum is denoted as $q$. The Feynman diagrams of the NTP processes in the SM are shown in Fig. 2.

\section{A. Experimental results}

The muonic NTP, $\nu_{\mu} \rightarrow \nu_{\mu} \mu \bar{\mu}$, has been measured by the CHARM-II [45], CCFR [46], and NuTeV [47] experiments. The results are given as the ratio of the observed cross section to the $\mathrm{SM}$ prediction, $\sigma_{\mathrm{SM}}$,

$$
\begin{aligned}
\frac{\sigma_{\mathrm{CHARM}-\mathrm{II}}}{\sigma_{\mathrm{SM}}} & =1.58 \pm 0.57, \\
\frac{\sigma_{\mathrm{CCFR}}}{\sigma_{\mathrm{SM}}} & =0.82 \pm 0.28, \\
\frac{\sigma_{\mathrm{NuTeV}}}{\sigma_{\mathrm{SM}}} & =0.72_{-0.72}^{+1.73} .
\end{aligned}
$$

The CHARM-II and CCFR results are consistent with the $\mathrm{SM}$ prediction within the error. The $\mathrm{NuTeV}$ result has relatively large uncertainty and includes a null result. Therefore we use the CHARM-II and CCFR results as constraints for our analyses.

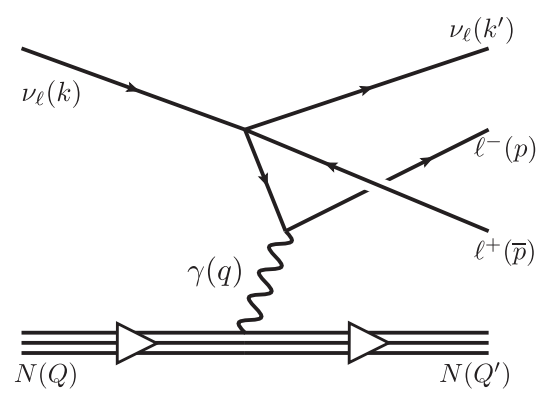

(b)

FIG. 2. Feynman diagrams of the NTP processes in the SM and four momentum assignment. 


\section{B. Amplitudes}

From Eq. (2), the SM amplitude of the NTP processes in Fig. 2 is given by

$$
\begin{aligned}
\mathcal{M}_{\mathrm{SM}}= & \frac{4 e^{2} G_{F}}{\sqrt{2}}\left[\overline{u_{\nu}}\left(k^{\prime}\right) \gamma^{\alpha} P_{L} u_{\nu}(k)\right]\left[\overline{u_{\ell}}(p) O_{\alpha}^{\mu} v_{\ell}(\bar{p})\right] \\
& \times \frac{1}{q^{2}}\left\langle Q^{\prime}\left|J_{\mu}\left(-q^{2}\right)\right| Q\right\rangle,
\end{aligned}
$$

where $u_{\ell}\left(v_{\ell}\right)$ and $u_{\nu}$ are the spinor of the charged (anti) lepton and neutrino, respectively. The operator $O_{\alpha}^{\mu}$ represents the charged lepton current part which is defined by

$$
\begin{aligned}
O_{\alpha}^{\mu}= & \gamma^{\mu} \frac{\not p+q+m_{\ell}}{(p+q)^{2}-m_{\ell}^{2}} \gamma_{\alpha}\left(g_{L} P_{L}+g_{R} P_{R}\right) \\
& +\gamma_{\alpha}\left(g_{L} P_{L}+g_{R} P_{R}\right) \frac{-\not p-q+m_{\ell}}{(\bar{p}+q)^{2}-m_{\ell}^{2}} \gamma^{\mu},
\end{aligned}
$$

where $m_{l}$ is the mass of the charged lepton. Throughout this paper, neutrinos are assumed to be massless. Note that $O_{\alpha}^{\mu}$ satisfies the current conservation condition, $q_{\mu} O_{\alpha}^{\mu}=0$ [38]. The operator $J_{\mu}$ in the bracket product is the electromagnetic current for the nucleus.

From Eqs. (9) and (10), the squared amplitude with summing over spins is obtained as

$$
\sum_{\text {spins }}\left|\mathcal{M}_{\mathrm{SM}}\right|^{2}=\frac{e^{4} G_{F}^{2}}{2 q^{4}} j^{\alpha \beta} L_{\alpha \beta}^{\mu \nu} J_{\mu \nu}
$$

where $j^{\alpha \beta}, L_{\alpha \beta}^{\mu \nu}$, and $J_{\mu \nu}$ represent neutrino, charged lepton, and nucleus contributions, respectively. These tensors are defined as

$$
\begin{aligned}
j^{\alpha \beta}= & 8\left(k^{\alpha} k^{\prime \beta}+k^{\beta} k^{\prime \alpha}-k \cdot k^{\prime} g^{\alpha \beta}-i \epsilon^{\rho \alpha \sigma \beta} k_{\rho} k_{\sigma}^{\prime}\right), \\
L_{\alpha \beta}^{\mu \nu}= & 4 \operatorname{Tr}\left[\left(\not p+m_{\ell}\right) W_{\alpha}^{\mu}\left(g_{L} P_{L}+g_{R} P_{R}\right)\left(\not \vec{p}-m_{\ell}\right)\right. \\
& \left.\times V_{\beta}^{\nu}\left(g_{L}^{*} P_{L}+g_{R}^{*} P_{R}\right)\right], \\
J_{\mu \nu}= & \left\langle Q^{\prime}\left|J_{\mu}(t)\right| Q\right\rangle\left\langle Q\left|J_{\nu}^{\dagger}(t)\right| Q^{\prime}\right\rangle,
\end{aligned}
$$

where $t$ is defined by $t=-q^{2}=-\left(Q^{\prime}-Q\right)^{2}$. For convenience, we express the contraction of three tensors as

$j^{\alpha \beta} L_{\alpha \beta}^{\mu \nu} J_{\mu \nu}=\left|g_{L}\right|^{2} M_{L}+\left|g_{R}\right|^{2} M_{R}-\left(g_{L} g_{R}^{*}+g_{L}^{*} g_{R}\right) M_{L R}$,

where the concrete forms of $M_{L}, M_{R}$, and $M_{L R}$ are given in Appendix A. Since the cross term of $g_{L}$ and $g_{R}, M_{L R}$, is proportional to $m_{\ell}^{2}$, it is subleading when the lepton mass $m_{\ell}$ is small compared to the energy scale of the NTP process. Each term in Eq. (13) is invariant under the exchange of the lepton momenta, $(p, k) \leftrightarrow\left(\bar{p}, k^{\prime}\right)$. Furthermore, under the exchange of either $p \leftrightarrow \bar{p}$ or $k \leftrightarrow k^{\prime}, M_{L}$ and $M_{R}$ are exchanged with each other,

$$
M_{L}^{\mu \nu} \leftrightarrow M_{R}^{\mu \nu},
$$

while $M_{L R}$ remains the same. The nucleus tensor, Eq. (12c), can be expressed in terms of a nuclear form factor. For the spin-0 nucleus,

$$
J_{\mu \nu}=Z^{2}\left(Q+Q^{\prime}\right)_{\mu}\left(Q+Q^{\prime}\right)_{\nu}|F(t)|^{2},
$$

where $Z$ is the atomic number of nucleus, $F(t)$ is the nuclear form factor given by

$$
F(t)=4 \pi \int_{0}^{\infty} d r r^{2} \rho(r) \frac{\sin \sqrt{t} r}{\sqrt{t} r},
$$

and $\rho(r)$ is the nuclear density. The normalization condition for $\rho(r)$ is

$$
4 \pi \int_{0}^{\infty} d r r^{2} \rho(r)=1
$$

and the integral variable $r$ is a distance from the center of the nucleus.

According to Refs. [51-53], the DUNE experiment will provide us with a large number of NTP events, where liquid argon is used at the near detector. In our numerical analysis, we consider argon to be the target nucleus. Following Ref. [58], we parametrize $\rho$ as

$$
\rho(r)=\rho_{0} \frac{1+w \frac{r^{2}}{c^{2}}}{1+\exp \left(\frac{r-c}{z}\right)},
$$

where $\rho_{0}$ is a normalization factor. The parameters are given as $c=3.73 \mathrm{fm}, z=0.62 \mathrm{fm}, w=-0.19$ for ${ }^{40} \mathrm{Ar}$. The $Z^{\prime}$ contribution to the NTP processes is shown in Fig. 3. The amplitude has the same spinor structure as Eq. (9). The only difference between the SM amplitude and the $Z^{\prime}$ amplitude is the propagation of $Z^{\prime}$ instead of $G_{F}$. Thus, the total amplitude squared of the NTP processes in

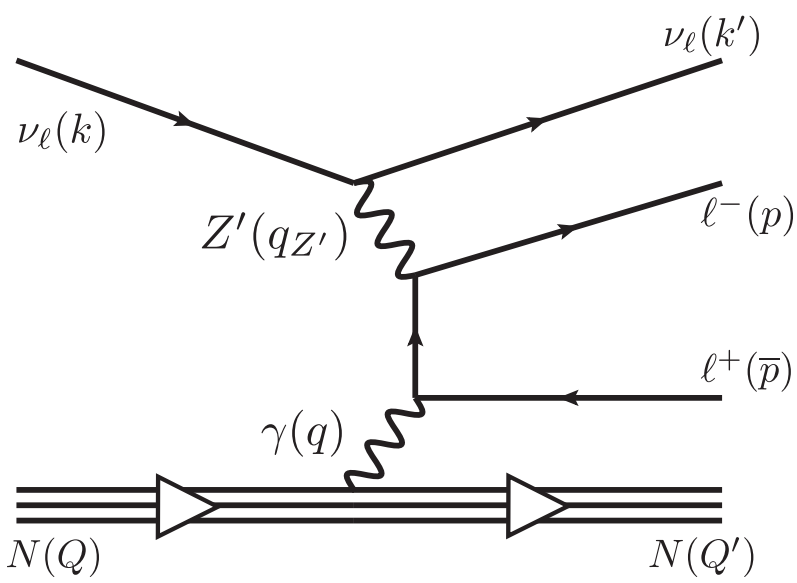

FIG. 3. The $Z^{\prime}$ contribution of the Feynman diagrams of NTP. 

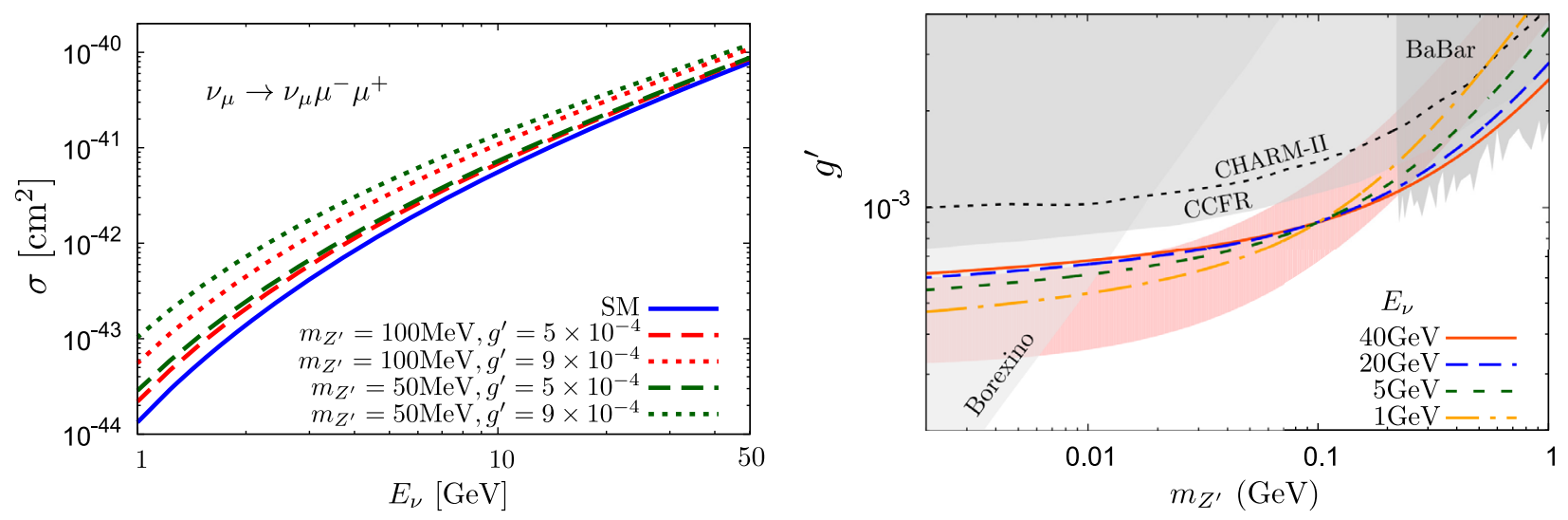

FIG. 4. Left: Incident neutrino beam energy dependence of the total cross section of $\nu_{\mu} \rightarrow \nu_{\mu} \mu \bar{\mu}$ in the $L_{\mu}-L_{\tau}$ model. The mass and gauge coupling constants to each curve are indicated in the figure. The blue curve represents the total cross section of the SM. Right: Contour plots of the total cross section in the $m_{Z^{\prime}} g^{\prime}$ plane. The values of the total cross section for $\left(m_{Z}^{\prime}, g^{\prime}\right)=\left(0.1 \mathrm{GeV}, 9 \times 10^{-4}\right)$ are given in Table II. The pink band represents the favored region of muon $g-2$ within $2 \sigma$, and the gray areas are the exclusion region by the Borexino, CHARM-II, CCFR, and BABAR experiments.

our model, $\left|\mathcal{M}_{\text {total }}\right|^{2}$, is obtained by simply replacing $g_{L}$ and $g_{R}$ in Eq. $(12 \mathrm{~b})$ as

$$
g_{L(R)} \rightarrow g_{L(R)} \mp \frac{\sqrt{2}}{4 G_{F}} \frac{g^{\prime 2}}{q_{Z^{\prime}}^{2}-m_{Z^{\prime}}^{2}},
$$

for $\nu_{\mu} \rightarrow \nu_{\mu} \mu \bar{\mu}(-)$ and $\nu_{\mu} \rightarrow \nu_{\mu} \tau \bar{\tau}(+)$. From Eq. (19), the NP parameter dependence disappears when

TABLE II. Parameters for the same value of the cross sections for $\nu_{\mu} \rightarrow \nu_{\mu} \mu \bar{\mu}$. The units of $E_{\nu}$ and $m_{Z^{\prime}}$ are $\mathrm{GeV}$, and that of $\sigma$ is $10^{-41} \mathrm{~cm}^{2}$, respectively. The first row for each $E_{\nu}$ is the cross section in the SM.

\begin{tabular}{cccc}
\hline \hline$E_{\nu}$ & $m_{Z^{\prime}}$ & $g^{\prime}$ & $\sigma$ \\
\hline 1 & $\ldots$ & $\ldots$ & $1.33 \times 10^{-3}$ \\
& 0.020 & $5.869 \times 10^{-4}$ & $5.54 \times 10^{-3}$ \\
& 0.10 & $9.000 \times 10^{-4}$ & $5.54 \times 10^{-3}$ \\
& 0.20 & $1.299 \times 10^{-3}$ & $5.54 \times 10^{-3}$ \\
& 1.0 & $4.972 \times 10^{-3}$ & $5.54 \times 10^{-3}$ \\
& $\ldots$ & $\ldots$ & $1.38 \times 10^{-1}$ \\
5 & 0.020 & $6.584 \times 10^{-4}$ & 0.328 \\
& 0.10 & $9.000 \times 10^{-4}$ & 0.328 \\
& 0.20 & $1.177 \times 10^{-3}$ & 0.328 \\
& 1.0 & $3.632 \times 10^{-3}$ & 0.328 \\
& $\ldots$ & $\ldots$ & 1.8945 \\
& 0.020 & $7.009 \times 10^{-4}$ & 3.097 \\
& 0.10 & $9.000 \times 10^{-4}$ & 3.097 \\
& 0.20 & $1.111 \times 10^{-3}$ & 3.097 \\
& 1.0 & $2.824 \times 10^{-3}$ & 3.097 \\
& $\ldots$ & $\ldots$ & 5.61 \\
& 0.020 & $7.18 \times 10^{-4}$ & 8.08 \\
& 0.10 & $9.000 \times 10^{-4}$ & 8.08 \\
& 0.20 & $1.084 \times 10^{-3}$ & 8.08 \\
& 1.0 & $2.513 \times 10^{-3}$ & 8.08 \\
\hline \hline
\end{tabular}

$\left|q_{Z^{\prime}}^{2}\right|+m_{Z^{\prime}}^{2} \gg \frac{\sqrt{2}}{4 G_{F}} g^{\prime 2} \simeq(157 \mathrm{MeV})^{2}\left(\frac{g^{\prime}}{9 \times 10^{-4}}\right)^{2}$.

As we will see in the next section, the $Z^{\prime}$ contribution is negligible in the tauonic NTP process. This fact suggests that $\left|q_{Z^{\prime}}^{2}\right|$ will become larger as the final state leptons become heavier. Then, the cross section is almost the same as that of the SM for the tauonic NTP process. In the next subsection, we show the dependence of the total cross section on the new physics parameters $g^{\prime}$ and $m_{Z^{\prime}}$.

\section{Trident production cross section}

The total cross section of the NTP is given by

$$
\sigma=\frac{1}{2\left(s-M^{2}\right)} \int d \Pi \sum_{\text {spins }}\left|\mathcal{M}_{\text {total }}\right|^{2},
$$

TABLE III. Parameters for the same value of the cross section for $\nu_{\mu} \rightarrow \nu_{\mu} \tau \bar{\tau}$. The units of $E_{\nu}$ and $m_{Z^{\prime}}$ are $\mathrm{GeV}$, and that of $\sigma$ is $10^{-47} \mathrm{~cm}^{2}$, respectively. The first row for each $E_{\nu}$ is the cross section in the SM.

\begin{tabular}{cccc}
\hline \hline$E_{\nu}$ & $m_{Z^{\prime}}$ & $g^{\prime}$ & $\sigma$ \\
\hline 10 & $\ldots$ & $\ldots$ & $1.94 \times 10^{-8}$ \\
& 0.020 & $9.53 \times 10^{-4}$ & $1.98 \times 10^{-8}$ \\
& 0.10 & $9.00 \times 10^{-4}$ & $1.96 \times 10^{-8}$ \\
& 0.20 & $9.29 \times 10^{-4}$ & $1.95 \times 10^{-8}$ \\
& 1.0 & $1.47 \times 10^{-3}$ & $1.94 \times 10^{-8}$ \\
40 & $\ldots$ & $\ldots$ & 1.87 \\
& 0.020 & $9.09 \times 10^{-4}$ & 1.87 \\
& 0.10 & $9.00 \times 10^{-4}$ & 1.87 \\
& 0.20 & $9.13 \times 10^{-4}$ & 1.87 \\
& 1.0 & $1.20 \times 10^{-3}$ & 1.87 \\
\hline \hline
\end{tabular}



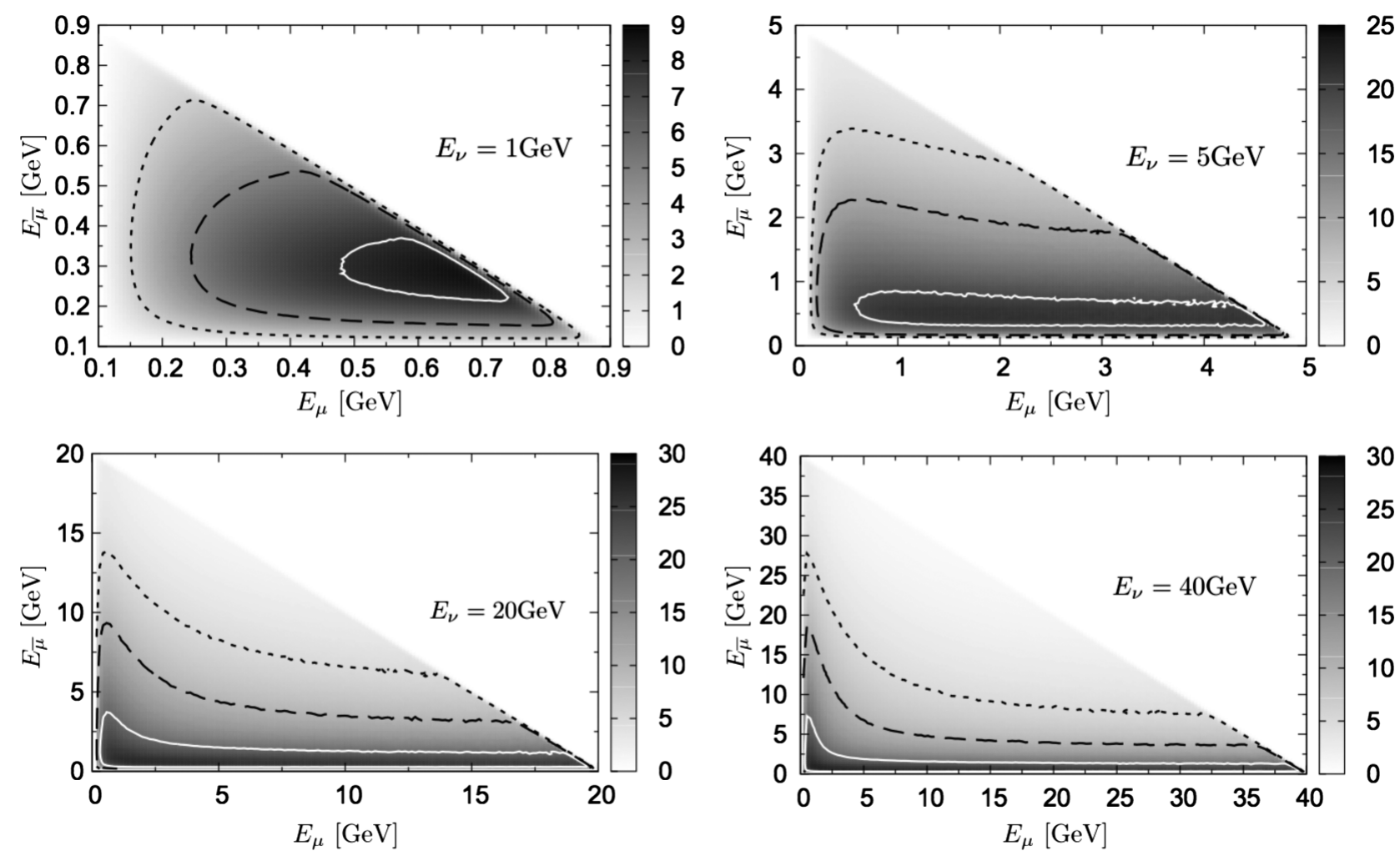

FIG. 5. Contour plots of the distribution in the $E_{\mu}-E_{\bar{\mu}}$ plane for $\nu_{\mu} \rightarrow \nu_{\mu} \mu \bar{\mu}$ in the SM. The color bar and contours are in the unit of $10^{-44} \mathrm{~cm}^{2} / \mathrm{GeV}^{2}$. The solid (white), dashed (black), and dotted (black) curves are the contours of 8,5, and $2 \mathrm{for} E_{\nu}=1 \mathrm{GeV}$, and 20,10, and 5 for $E_{\nu}=5,20,40 \mathrm{GeV}$, respectively.

where $s=(k+Q)^{2}$ is the center of mass energy and $d \Pi$ is the phase space integral measure given by

$$
\begin{aligned}
d \Pi= & \frac{d^{3} k^{\prime}}{(2 \pi)^{3} 2 E_{k^{\prime}}} \frac{d^{3} p}{(2 \pi)^{3} 2 E_{p}} \frac{d^{3} \bar{p}}{(2 \pi)^{3} 2 E_{\bar{p}}} \frac{d^{3} Q^{\prime}}{(2 \pi)^{3} 2 E_{Q^{\prime}}}(2 \pi)^{4} \\
& \times \delta^{(4)}\left(k^{\prime}+p+\bar{p}+Q^{\prime}-k-Q\right) .
\end{aligned}
$$

By the energy-momentum conservations and rotational symmetry, the number of integrals can be reduced from twelve to seven. Then, we perform the phase space integrations numerically using the changes of the integral variables shown in Appendix B.

The cross sections of the muonic NTP in the minimal $L_{\mu}-L_{\tau}$ model are shown in Fig. 4 . The left panel shows the cross sections with $m_{Z^{\prime}}=50,100 \mathrm{MeV}$ and $g^{\prime}=5 \times 10^{-4}$, $9 \times 10^{-4}$ as a function of the incident neutrino energy $E_{\nu}$. The right panel shows the contours of the muonic NTP cross section in our model. In the left panel, the parameters $\left(m_{Z^{\prime}}, g^{\prime}\right)$ are taken from the right panel as illustrating examples. The red curves correspond to $m_{Z^{\prime}}=100 \mathrm{MeV}$ and the green ones to $50 \mathrm{MeV}$, respectively. For comparison, we also show the cross section in the SM with a blue solid curve. Our results of the total cross section in the SM are in good agreement with previous studies [41,42] and [48,51]. It can be seen that the NP contributions become smaller when compared with the SM cross section as $E_{\nu}$ becomes higher. This behavior generally holds even for $Z^{\prime}$ with a much lighter mass than $E_{\nu}$. As we explained in the previous subsection, this is because $\left|q_{Z^{\prime}}^{2}\right|$ can take larger values than $m_{Z^{\prime}}^{2}$ for higher $E_{\nu}$; the NP contribution or the propagator of $Z^{\prime}$ decreases as $g^{\prime 2} /\left(G_{F} q_{Z^{\prime}}^{2}\right)$. Thus, the cross section is less sensitive to the NP parameters for higher $E_{\nu}$, which has been shown in Ref. [15]. Higher resolutions on momentum and/or energy measurements are required to solve the degeneracy in higher beam experiments like DUNE. On the other hand, for smaller $E_{\nu}$, the NP contributions to the cross section become larger, but the cross section itself becomes smaller. For example, for $E_{\nu}=1 \mathrm{GeV}$, the cross section is $\mathcal{O}\left(10^{-44}\right) \mathrm{cm}^{2}$.

In the right panel of Fig. 4, the red, blue, green, and orange curves correspond to the same cross sections for $E_{\nu}=40,20,5$, and $1 \mathrm{GeV}$, respectively. We chose $\left(m_{Z}^{\prime}, g^{\prime}\right)=\left(0.1 \mathrm{GeV}, 9 \times 10^{-4}\right)$ as a reference parameter set to determine the values of the cross section. Thus all curves intersect at this point. The pink band represents the muon $g-2$ favored region within $2 \sigma$ and the gray shaded regions are excluded by Borexino [15], ${ }^{1}$ CHARM-II [45],

\footnotetext{
${ }^{1}$ The constraints from Borexino are discussed in [59-61] in various different scenarios of new force. The constraint is translated from a $B-L$ gauge symmetric model in [15].
} 

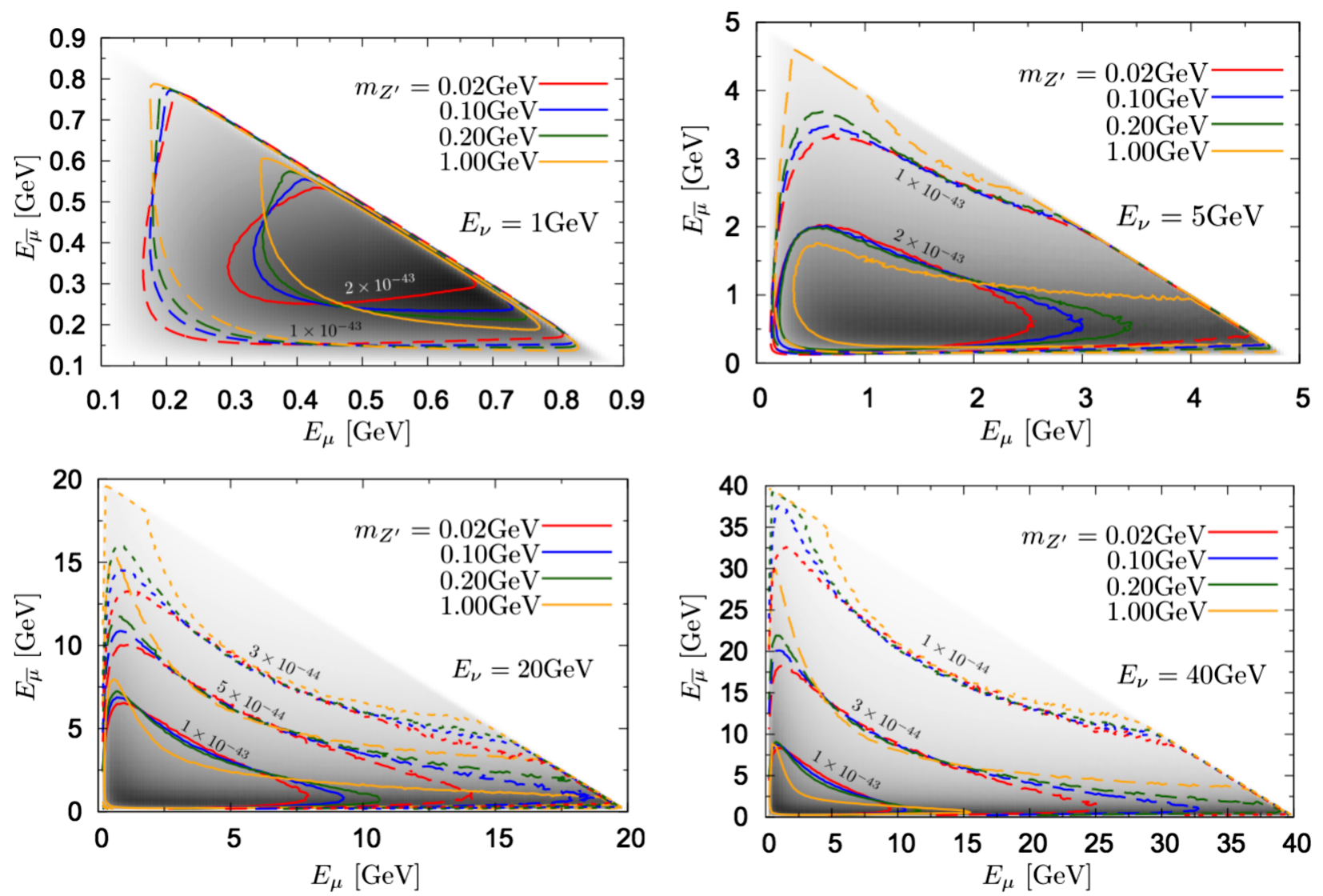

FIG. 6. Contour plots of the deviation from the SM in the $E_{\mu}-E_{\bar{\mu}}$ distribution of $\nu_{\mu} \rightarrow \nu_{\mu} \mu \bar{\mu}$. The red, blue, green, and orange curves correspond to the parameter sets in Table II. The solid, dashed, and dotted curves are the contours of $R\left(E_{\mu}, E_{\bar{\mu}}\right)$ with their values indicated near each curve. The background gray color is the SM distribution shown in Fig. 5.

CCFR [46], ${ }^{2}$ and $B A B A R[62]$. This plot clearly shows that the cross section is degenerate in $m_{Z^{\prime}}$ and $g^{\prime}$ over a wide range. As we mentioned in the Introduction, for the determination of the parameters, one needs additional information besides the cross section value.

For this purpose, we analyze the distributions in the energies, opening angle, and invariant mass of the final state charged leptons in the next section. The analyses are performed on the parameter sets shown in Table II for the muonic trident. The first row for each $E_{\nu}$ in Table II is the trident cross section in the SM. We chose $\left(m_{Z^{\prime}}, g^{\prime}\right)=$ $\left(0.1 \mathrm{GeV}, 9 \times 10^{-4}\right)$ as a reference parameter, which can explain $(g-2)_{\mu}$ within $2 \sigma$. Other parameter sets are chosen so that the cross sections have the same values with that of the reference set for each $E_{\nu}$. Note that some parameter sets are outside the $2 \sigma$ region of $(g-2)_{\mu}$ or in the gray region. However, we include those parameter sets to see the behavior of the distributions for comparison.

We also show the cross sections of tauonic NTP, $\nu_{\mu} \rightarrow \nu_{\mu} \tau \bar{\tau}$, in Table III for $E_{\nu}=10$ and $40 \mathrm{GeV}$. One finds that the cross sections for the reference point are

\footnotetext{
${ }^{2}$ The exclusion regions by CHARM-II and CCFR are taken from [13].
}

almost the same as those in the SM. This suggests that the new physics contributions are very small. For a tauonic trident to occur, the momentum transfer $\left|q_{Z^{\prime}}^{2}\right|$ will be of order $m_{\tau}^{2}$ and hence the new physics contribution is much suppressed, as shown in Eq. (20). In fact, we have performed the same analyses for the tauonic NTP as for the muonic one in the next section, and found that the distributions show tiny differences among the NP parameter sets in Table III. Therefore, we show our numerical results only for the muonic NTP in the next section.

\section{NUMERICAL RESULTS}

We show the distributions of the energies $E_{\mu}$ and $E_{\bar{\mu}}$, invariant mass $m_{\mu \bar{\mu}}^{2}$ and opening angle $\theta_{\mu \bar{\mu}}$ of the muon and antimuon in the SM and our model for the parameters given in Table II. To obtain the total cross section of the NTP processes, we have to perform the phase space integral over seven variables. For such high-dimensional integrals, the Monte Carlo integration is known to be useful due to its quick convergence compared to quadratures by parts.

To investigate the NP effect in the charged lepton distributions, we calculate the differential cross section with respect to some observables. In general, it is 

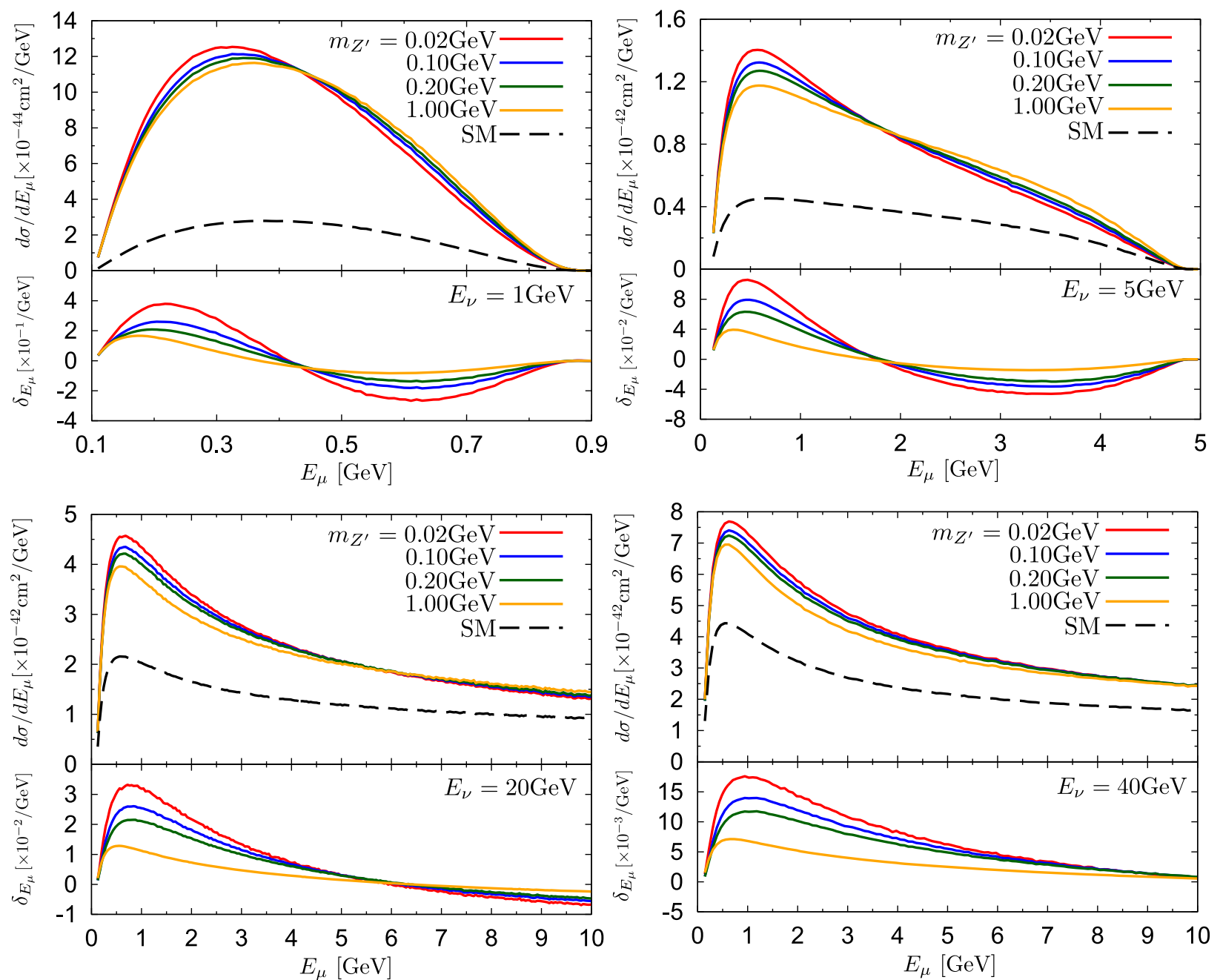

FIG. 7. The $E_{\mu}$ distribution (upper panel) and the shape of the distribution in the $L_{\mu}-L_{\tau}$ model. The color of the curves are the same as in Fig. 6. The black, dashed curve represents the SM distribution.

complicated to select an arbitrary observable as one of integral variables. However, when we use the Monte Carlo integration, we do not need to make the complicated variable transformation to obtain the differential cross section with respect to the favored observable.

Let $f(\boldsymbol{y})$ be a function of variables $\boldsymbol{y}$, which satisfies $\sigma=\int d \boldsymbol{y} f(\boldsymbol{y})$. Here, treating $\boldsymbol{y}$ as integral variables, we consider performing the Monte Carlo integration. To obtain $d \sigma / d x$, we prepare discretized bins of a variable $x$, which are labeled by $a$ and have an interval $\Delta x_{a}$. In this integration, we sample the variables $\boldsymbol{y}$ from the uniform probability distribution $N$ times. At the $i$-th step of the sampling, $x_{i}$ is calculated as well as $f\left(\boldsymbol{y}_{i}\right)$ for the generated $\boldsymbol{y}_{i}$. Then, one can approximate the distribution in the variable $x$ by

$$
\begin{aligned}
\frac{d \sigma}{d x}\left(x_{a}\right) \simeq & \frac{D}{N \Delta x_{a}} \sum_{i=1}^{N} f\left(\boldsymbol{y}_{i}\right) \theta\left(x_{i}-x_{a}+\frac{\Delta x_{a}}{2}\right) \\
& \times \theta\left(x_{a}+\frac{\Delta x_{a}}{2}-x_{i}\right),
\end{aligned}
$$

where $D$ is the total width of the $x$ bins and $N$ is the number of samples. The function $\theta(z)$ is a step function, which is a unity for $z \geq 0$ and zero for $z<0$. The total cross section can be obtained by summing Eq. (23) over $x$ as

$$
\sigma \simeq \sum_{a} \Delta x_{a} \frac{d \sigma}{d x}\left(x_{a}\right)
$$

\section{A. Energy distributions}

First, we show the SM distributions of $E_{\mu}$ and $E_{\bar{\mu}}$ for the process of $\nu_{\mu} \rightarrow \nu_{\mu} \mu \bar{\mu}$ in Fig. 5. The energy of the incoming neutrino is taken to be $E_{\nu}=1,5,20$, and $40 \mathrm{GeV}$, respectively. The color bar on the right indicates the value of the double differential cross section in unit of $10^{-44} \mathrm{~cm}^{2} / \mathrm{GeV}^{2}$. The solid (white), dashed (black), and dotted (black) curves are the contours of 8,5, and 2 in $10^{-44} \mathrm{~cm}^{2} / \mathrm{GeV}^{2}$ for $E_{\nu}=1 \mathrm{GeV}$, and 20,10 , and 5 in $10^{-44} \mathrm{~cm}^{2} / \mathrm{GeV}^{2}$ for $E_{\nu}=5,20,40 \mathrm{GeV}$, respectively.

In each panel, one can see that the distribution has a peak near the kinematical edge for $E_{\nu}=1 \mathrm{GeV}$. As $E_{\nu}$ becomes 

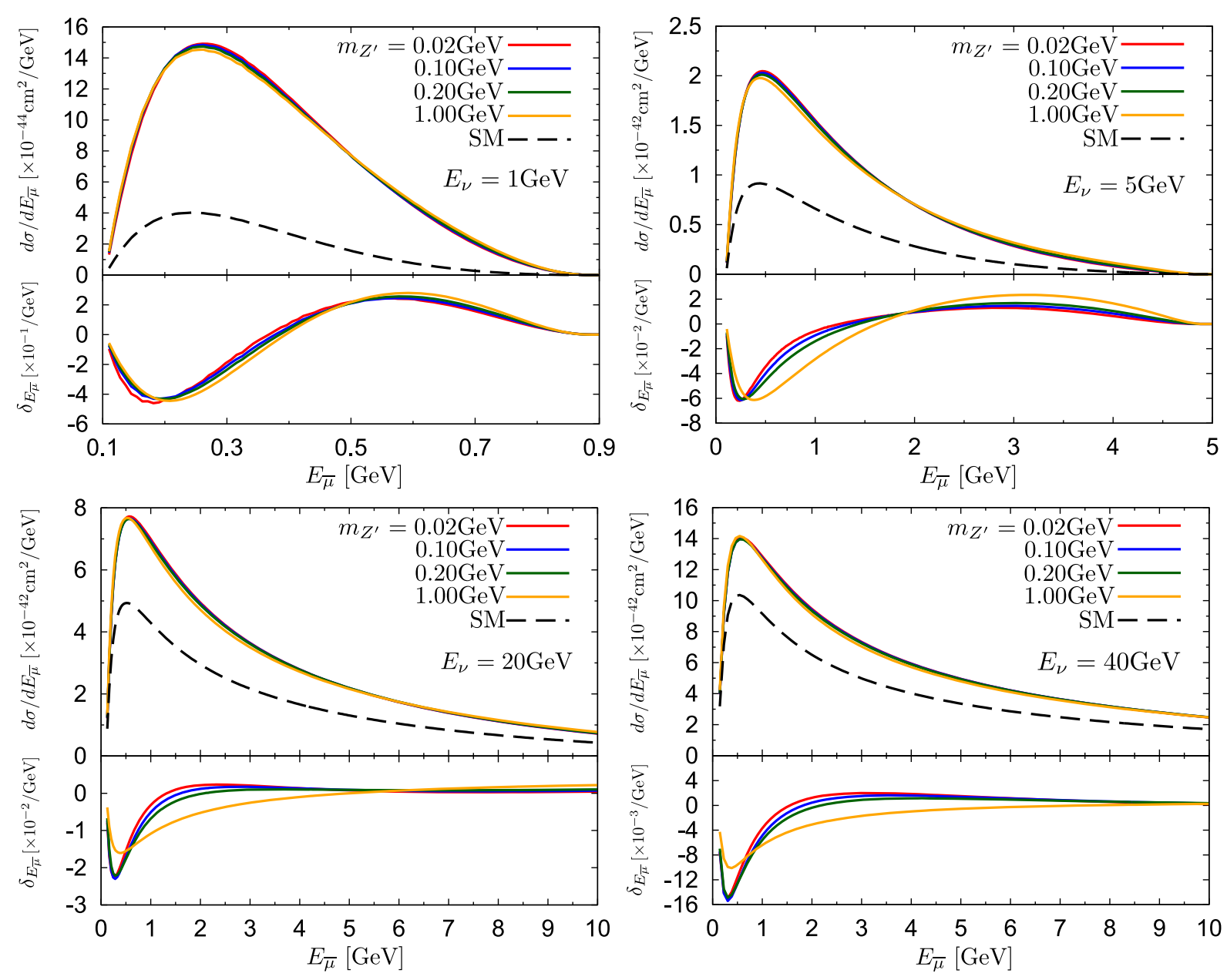

FIG. 8. $E_{\bar{\mu}}$ distributions in the $L_{\mu}-L_{\tau}$ model.

higher, the peak moves to the lower $E_{\mu}$ region. For $E_{\nu}>5 \mathrm{GeV}, E_{\mu}$ is more uniformly distributed than $E_{\bar{\mu}}$ is. We can understand this asymmetry of the distribution in the $E_{\mu}-E_{\bar{\mu}}$ plane as follows: As we explained in Eq. (14), the terms $M_{L}$ and $M_{R}$ in the lepton tensor are exchanged under $p \leftrightarrow \bar{p}$. Thus, the double differential cross section differs under the exchange of $E_{\mu} \leftrightarrow E_{\bar{\mu}}$ if the coupling constants $g_{L}$ and $g_{R}$ are different as in the SM. It should be noticed that the distribution becomes symmetric in the $E_{\mu}-E_{\bar{\mu}}$ plane for the case of $g_{L}=g_{R}$, such that the $L_{\mu}-L_{\tau}$ contributions dominate over the SM couplings.

To see the parameter dependence of the NP model, we show the deviation of the differential cross section in our model from the SM, which is defined by

$$
R\left(E_{\mu}, E_{\bar{\mu}}\right) \equiv \frac{d^{2} \sigma}{d E_{\mu} d E_{\bar{\mu}}}-\frac{d^{2} \sigma_{\mathrm{SM}}}{d E_{\mu} d E_{\bar{\mu}}} .
$$

In Fig. 6, the solid, dashed, and dotted curves are the contours of $R\left(E_{\mu}, E_{\bar{\mu}}\right)$ while the red, blue, green, and orange colors represent the parameter sets in Table II. In each panel, the values of $R\left(E_{\mu}, E_{\bar{\mu}}\right)$ are indicated near each curve, and only the $Z^{\prime}$ mass is shown to specify the parameter sets. The gray background represents the SM distribution shown in Fig. 5.

In Fig. 6, the parameter dependence can be seen clearly in the contours of $R\left(E_{\mu}, E_{\bar{\mu}}\right)$ in the high $E_{\mu}$ or $E_{\bar{\mu}}$ region. The contours extend to larger values of $E_{\mu}$ or $E_{\bar{\mu}}$ as the $Z^{\prime}$ mass becomes heavier. It is also shown that $E_{\mu}$ is more uniformly distributed than $E_{\bar{\mu}}$, due to the interference between the NP and SM contributions. We note that $R\left(E_{\mu}, E_{\bar{\mu}}\right)$ is positive for all $\left(E_{\mu}, E_{\bar{\mu}}\right)$ in $\nu_{\mu} \rightarrow \nu_{\mu} \mu \bar{\mu}$ in our calculation. The $L_{\mu}-L_{\tau}$ contribution enhances $\nu_{\mu} \rightarrow$ $\nu_{\mu} \mu \bar{\mu}$ because both $g_{L}$ and $g_{R}$ are effectively enlarged by the propagator of the $Z^{\prime}$ boson as seen in Eq. (19).

Figures 7 and 8 show that the distributions (upper panel) and difference of shape of the distribution from the SM (lower panel) in $E_{\mu}$ and $E_{\bar{\mu}}$, respectively. The colors of the solid curves are the same as in Fig. 6 and the black dashed curve is the SM distribution. In the upper panels of Fig. 7, the parameter dependence of the distributions can be seen in two regions: around the peaks in the lower $E_{\mu}$ and at the tails in the higher $E_{\mu}$. The parameter dependence is clearer 

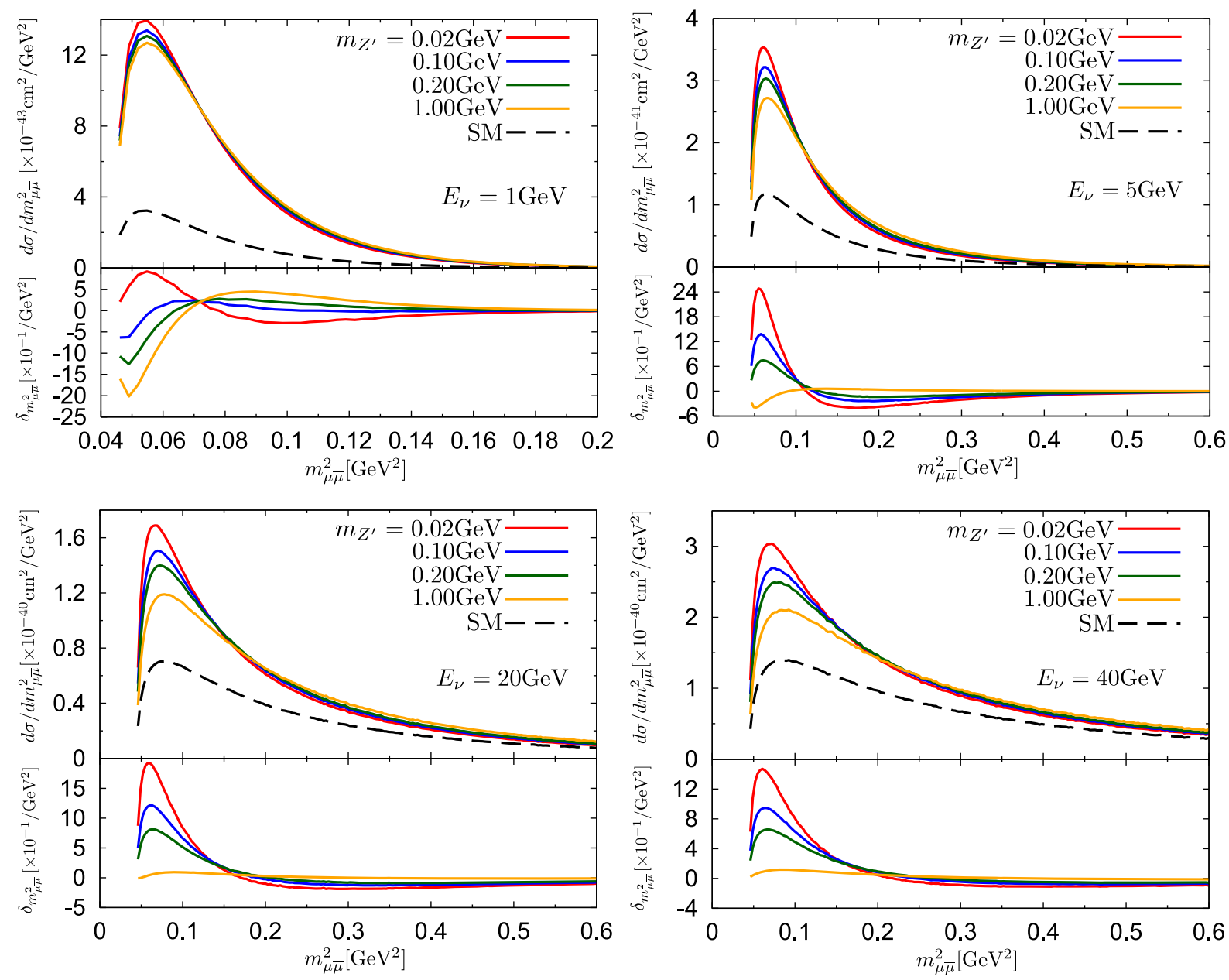

FIG. 9. $\quad m_{\mu \bar{\mu}}^{2}$ distributions in the $L_{\mu}-L_{\tau}$ model.

around the peaks than at the tails. In each panel, one can see that the peaks become higher as the $Z^{\prime}$ mass becomes lighter. It is also seen that the $E_{\mu}$ corresponding to the peak slightly differs among the parameter sets in each energy. On the other hand, in the upper panels of Fig. 8, the $E_{\bar{\mu}}$ distribution is less dependent on the parameters compared to the $E_{\mu}$ distributions. These results imply that the $E_{\mu}$ distribution is more useful to determine the new physics parameters.

To see how the NP contributions modify the shape of the distributions, we define

$$
\delta_{X} \equiv \frac{1}{\sigma} \frac{d \sigma}{d X}-\frac{1}{\sigma_{\mathrm{SM}}} \frac{d \sigma_{\mathrm{SM}}}{d X},
$$

for an arbitrary kinematical variable $X$. In the lower panels of Figs. 7 and 8, we plotted $\delta_{E_{\mu}}$ and $\delta_{E_{\tilde{\mu}}}$, respectively. These are the difference of the normalized distributions, and become zero when the shape of the distributions are the same between our model and the SM, even if overall magnitudes are different. One can see in Fig. 7 that $\delta_{E_{\mu}}$ is positive in the lower $E_{\mu}$ and negative in the higher $E_{\mu}$ for all parameter sets. On the other hand, in Fig. $8, \delta_{E_{\bar{\mu}}}$ shows the opposite behavior. Thus, the distributions are shifted to the lower $E_{\mu}$ and the higher $E_{\bar{\mu}}$ by the NP contribution. The shape of the distribution also depends on the NP parameter set. In the $E_{\mu}$ distribution, $\left|\delta_{E_{\mu}}\right|$ is larger for the lighter $Z^{\prime}$ mass. Such information can be used to determine the parameters.

\section{B. Invariant mass distributions}

The invariant mass of the outgoing muon and antimuon is defined by

$$
m_{\mu \bar{\mu}}^{2} \equiv(p+\bar{p})^{2} .
$$

In Fig. 9, we show the invariant mass distribution (upper panel) and the shape difference of this distribution from the SM (lower panel).

We can see from each panel that the distribution clearly depends on the parameters in the lower value of the $m_{\mu \bar{\mu}}^{2}$ region. The peaks of the distributions become sharper as the $Z^{\prime}$ mass is lighter and the dependence becomes more 

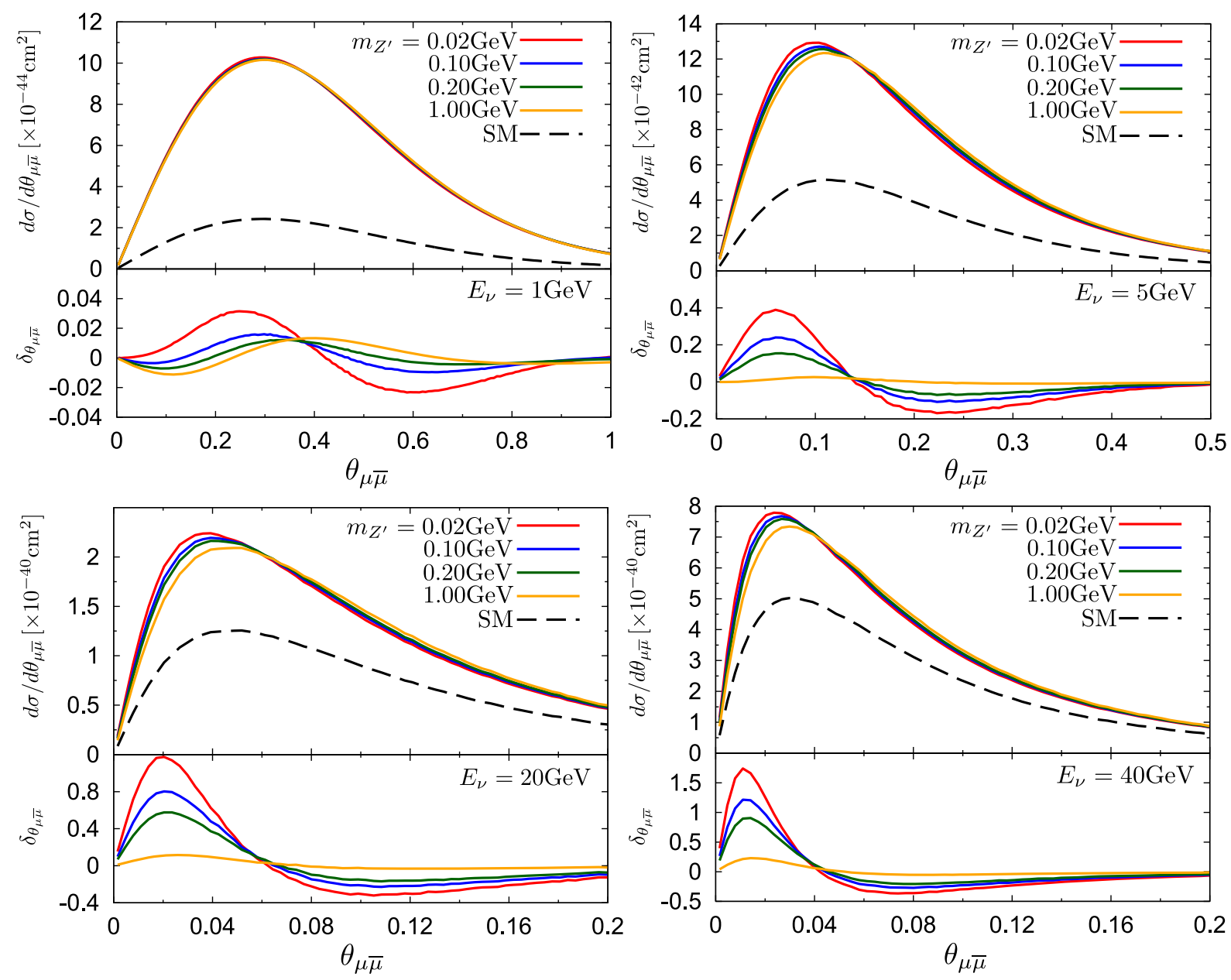

FIG. 10. Muon opening angle distributions of the cross section in the $L_{\mu}-L_{\tau}$ model.

significant as $E_{\nu}$ is higher. It is shown that the $m_{\mu \bar{\mu}}^{2}$ corresponds to the peak changes for the parameter sets. From the lower panels, one can also see that $\delta_{m_{\mu \mu}^{2}}$, defined by Eq. (26), can take positive and negative values in the lower value of $m_{\mu \bar{\mu}}^{2}$ depending on the parameters, which shows different behaviors from the energy distributions.

\section{Opening angle distributions}

The opening angle of the outgoing muon and antimuon can be defined by

$$
\cos \theta_{\mu \bar{\mu}} \equiv \frac{\boldsymbol{p} \cdot \overline{\boldsymbol{p}}}{|\boldsymbol{p}||\overline{\boldsymbol{p}}|}
$$

where $\boldsymbol{p}$ and $\overline{\boldsymbol{p}}$ are the momenta of the muon and antimuon, respectively. Figure 10 shows the distributions of $\theta_{\mu \bar{\mu}}$ (upper panel) and the shape (lower panel). From this figure, one finds that the opening angle distributions show the parameter dependences around the peaks for the higher $E_{\nu}$. It is also seen that the angle for the peaks becomes smaller as $E_{\nu}$ becomes higher. Similar behavior can be seen in the shape of the distributions.
In this analysis, we have considered only the coherent NTP processes assuming argon as a target material. Since the kinematical distributions depend on the form factor, the nuclear dependence is worth investigating for finding the best target material to identify the NP parameters.

We also understand that the diffractive NTP processes can be relevant for the available neutrino energy in experiments. We should check whether the diffractive contribution causes positive or negative effects for the measurement of NP parameters. We will study these topics in our future works.

Before closing this section, we comment about the SM background events to the muonic NTP process. The main background for the muonic NTP consists of chargedcurrent, single-pion production events, $\nu_{\mu} N \rightarrow \mu \pi N^{\prime}$, in which the muon and pion tracks can be misidentified. Such backgrounds were studied for DUNE in [51-53]. It was shown that the number of the total background events are comparable to or larger than those of the signal events, which can be reduced by applying kinematical cuts for the invariant mass and angle of the muons. These backgrounds would affect the resolution for the determination of the new physics parameters and therefore should be taken into account. 


\section{SUMMARY}

We have considered the minimal gauged $L_{\mu}-L_{\tau}$ model, and studied the dependences of the distributions of the neutrino trident production process on the new physics parameters, $m_{Z^{\prime}}$ and $g^{\prime}$. We analyzed the distributions of energies, opening angle, and invariant mass of muons and their shapes in $\nu_{\mu} \rightarrow \nu_{\mu} \mu \bar{\mu}$.

We have found that the distributions can be different among the NP parameter sets for which the total cross section is the same. In $E_{\mu}-E_{\bar{\mu}}$ distributions, the differences can be seen in larger $E_{\mu}$ or $E_{\bar{\mu}}$ regions. We also found that the parameter dependences in the $E_{\mu}$ and $m_{\mu \bar{\mu}}^{2}$ distributions are clearer compared with those in the $E_{\bar{\mu}}$ and $\theta_{\mu \bar{\mu}}$ distributions. Therefore the $E_{\mu}$ and $m_{\mu \bar{\mu}}^{2}$ distributions will be useful to determine the NP parameters. The shapes of the distributions were also presented, which shows the parameter dependence.

The determination of the new physics parameters by combining the information of the total cross section and distributions will be the next step of our work. Such a study will need more detailed information including a resolution, efficiencies, and SM backgrounds in experiments. We leave such analyses for the next work.

\section{ACKNOWLEDGMENTS}

This work is supported by JSPS KAKENHI Grant No. JP18K03651 and MEXT KAKENHI Grant No. JP18H05543 (T. S.), the Sasakawa Scientific Research Grant from the Japan Science Society (Y. U.), and JSPS KAKENHI Grant No. JP18H01210 (T. S. and Y. U.).

\section{APPENDIX A: LEPTON TENSOR}

In this Appendix, we present the analytic formula of the amplitude squared for $\nu_{\mu} \rightarrow \nu_{\mu} \ell \bar{\ell}$. The lepton and nucleus parts are written as a neutrino tensor $j^{\alpha \beta}$, charged lepton tensor $L_{\alpha \beta}^{\mu \nu}$, and nucleus tensor $J_{\mu \nu}$. Then, the amplitude squared is given by $j^{\alpha \beta} L_{\alpha \beta}^{\mu \nu} J_{\mu \nu}$ up to overall factors. Using the expression of the charged lepton tensor, (12b), it can be classified by the chirality of outgoing charged leptons as

$j^{\alpha \beta} L_{\alpha \beta}^{\mu \nu} J_{\mu \nu}=\left|g_{L}\right|^{2} M_{L}+\left|g_{R}\right|^{2} M_{R}-g_{L} g_{R}^{*} M_{L R}-g_{L}^{*} g_{R} M_{R L}$,

where

$$
\begin{aligned}
& M_{L}=4 J_{\mu \nu} j^{\alpha \beta} \operatorname{Tr}\left[\not p W_{\alpha}^{\mu} \bar{p} V_{\beta}^{\nu} P_{L}\right], \\
& M_{R}=4 J_{\mu \nu} j^{\alpha \beta} \operatorname{Tr}\left[\not \not W_{\alpha}^{\mu} \bar{p} V_{\beta}^{\nu} P_{R}\right], \\
& M_{L R}=4 m_{\ell}^{2} J_{\mu \nu} j^{\alpha \beta} \operatorname{Tr}\left[W_{\alpha}^{\mu} V_{\beta}^{\nu} P_{R}\right], \\
& M_{R L}=4 m_{\ell}^{2} J_{\mu \nu} j^{\alpha \beta} \operatorname{Tr}\left[W_{\alpha}^{\mu} V_{\beta}^{\nu} P_{L}\right] .
\end{aligned}
$$

Here, $W_{\alpha}^{\mu}$ and $V_{\beta}^{\nu}$ are the propagators of leptons given by

$$
W_{\alpha}^{\mu}=\frac{2 p^{\mu}+\gamma^{\mu} \not \phi}{q^{2}+2 p \cdot q} \gamma_{\alpha}-\gamma_{\alpha} \frac{2 \bar{p}^{\mu}+\not q \gamma^{\mu}}{q^{2}+2 \bar{p} \cdot q},
$$

and

$V_{\beta}^{\nu}=\gamma_{0} W_{\beta}^{\dagger \nu} \gamma_{0}=\gamma_{\beta} \frac{2 p^{\nu}+\not \gamma^{\nu}}{q^{2}+2 p \cdot q}-\frac{2 \bar{p}^{\nu}+\gamma^{\nu} \not}{q^{2}+2 \bar{p} \cdot q} \gamma_{\beta}$.

Due to the parity conservation of the electromagnetic interaction, $J_{\mu \nu}$ must be symmetric with respect to $\mu$ and $\nu$, regardless of the detail of the nucleus. Thus, it is enough to calculate the symmetric part of $j^{\alpha \beta} L_{\alpha \beta}^{\mu \nu}$ under $\mu \leftrightarrow \nu$. The concrete form of the nucleus tensor $J_{\mu \nu}$ is determined by the target nucleus, as given in Eq. (15) with (18).

By straightforward calculation, we obtain the explicit formula for $M_{L}$ in terms of lepton momenta as follows:

$$
\begin{gathered}
M_{L}=\left(-256 J_{\mu \nu}\right)\left\{\frac{M_{L 1}^{\mu \nu}}{\left(q^{2}+2 p \cdot q\right)^{2}}+\frac{M_{L 2}^{\mu \nu}}{\left(q^{2}+2 \bar{p} \cdot q\right)^{2}}+\frac{M_{L 3}^{\mu \nu}}{\left(q^{2}+2 p \cdot q\right)\left(q^{2}+2 \bar{p} \cdot q\right)}\right\}, \\
M_{L 1}^{\mu \nu}=k \cdot \bar{p}\left[g^{\mu \nu}\left(2 q \cdot k^{\prime} q \cdot p-q^{2} k^{\prime} \cdot p\right)+\left(p^{\mu} k^{\prime \nu}+p^{\nu} k^{\prime \mu}\right)\left(q^{2}+2 p \cdot q\right)\right. \\
\left.-2\left(p^{\mu} q^{\nu}+p^{\nu} q^{\mu}+2 p^{\mu} p^{\nu}\right)\left\{k^{\prime} \cdot(p+q)\right\}\right], \\
M_{L 2}^{\mu \nu}=\left(\{p, k\} \leftrightarrow\left\{\bar{p}, k^{\prime}\right\} \text { exchange of } M_{L 1}^{\mu \nu}\right),
\end{gathered}
$$




$$
\begin{aligned}
M_{L 3}^{\mu \nu}= & 2 g^{\mu \nu}\left\{p \cdot q\left(k^{\prime} \cdot \bar{p} q \cdot k-q \cdot \bar{p} k \cdot k^{\prime}\right)+q \cdot k^{\prime}(q \cdot \bar{p} k \cdot p-p \cdot \bar{p} q \cdot k)+q^{2}\left(p \cdot \bar{p} k \cdot k^{\prime}-p \cdot k \bar{p} \cdot k^{\prime}\right)\right\} \\
& +\left(p^{\mu} \bar{p}^{\nu}+p^{\nu} \bar{p}^{\mu}\right)\left(4 k \cdot \bar{p} k^{\prime} \cdot p+2 k \cdot q k^{\prime} \cdot q-q^{2} k \cdot k^{\prime}+2 k \cdot \bar{p} q \cdot k^{\prime}+2 k^{\prime} \cdot p q \cdot k\right) \\
& +\left(p^{\mu} q^{\nu}+p^{\nu} q^{\mu}\right)\left(k \cdot \bar{p} q \cdot k^{\prime}+q \cdot \bar{p} k \cdot k^{\prime}-q \cdot k k^{\prime} \cdot \bar{p}+2 k^{\prime} \cdot p k \cdot \bar{p}\right) \\
& +\left(\bar{p}^{\mu} q^{\nu}+\bar{p}^{\nu} q^{\mu}\right)\left(k^{\prime} \cdot p q \cdot k+q \cdot p k \cdot k^{\prime}-q \cdot k^{\prime} k \cdot p+2 k \cdot \bar{p} k^{\prime} \cdot p\right) \\
& -\left(p^{\mu} k^{\nu}+p^{\nu} k^{\mu}\right)\left(2 k^{\prime} \cdot p q \cdot \bar{p}+2 q \cdot \bar{p} q \cdot k^{\prime}-q^{2} k^{\prime} \cdot \bar{p}\right)-\left(\bar{p}^{\mu} k^{\prime \nu}+\bar{p}^{\nu} k^{\prime \mu}\right)\left(2 k \cdot \bar{p} q \cdot p+2 q \cdot p q \cdot k-q^{2} k \cdot p\right) \\
& +\left(q^{\mu} k^{\nu}+q^{\nu} k^{\mu}\right)\left(p \cdot \bar{p} q \cdot k^{\prime}-k^{\prime} \cdot p q \cdot \bar{p}-k^{\prime} \cdot \bar{p} q \cdot p\right)+\left(q^{\mu} k^{\prime \nu}+q^{\nu} k^{\prime \mu}\right)(p \cdot \bar{p} q \cdot k-k \cdot \bar{p} q \cdot p-k \cdot p q \cdot \bar{p}) \\
& +\left(k^{\mu} k^{\prime \nu}+k^{\nu} k^{\prime \mu}\right)\left(2 q \cdot p q \cdot \bar{p}-q^{2} p \cdot \bar{p}\right)+\left(q^{\mu} q^{\nu}+q^{\nu} q^{\mu}\right)\left(k^{\prime} \cdot \bar{p} k \cdot p-k \cdot k^{\prime} p \cdot \bar{p}+k^{\prime} \cdot p k \cdot \bar{p}\right) .
\end{aligned}
$$

In the case of $V-A$ interaction, the amplitude squared is only $M_{L}$ which was given in [38].

Next, moving on to the explicit form of $M_{R}$, one can easily derive it by taking the charge conjugate of materials in the trace

$$
\begin{aligned}
M_{R} & =4 J_{\mu \nu} j^{\alpha \beta} \operatorname{Tr}\left[\not p W_{\beta}^{\nu} \not \bar{p} V_{\alpha}^{\mu} P_{L}\right] \\
& =4 J_{\mu \nu} j^{\beta \alpha} \operatorname{Tr}\left[\not p W_{\alpha}^{\mu} \bar{p} V_{\beta}^{\nu} P_{L}\right] .
\end{aligned}
$$

One notices that the form in the last line is the same as that of $M_{L}$ except for the superscripts of the neutrino tensor $j^{\alpha \beta}$. According to Eq. (12), the exchange of $\mu$ and $\nu$ in $j^{\mu \nu}$ clearly corresponds to the exchange of $k$ and $k^{\prime}$. Therefore, $M_{R}$ is obtained as

$$
M_{R}=\left(k \leftrightarrow k^{\prime} \text { exchange of } M_{L}\right) .
$$

At last, we present $M_{L R}$ and $M_{R L}$. By using the explicit forms of $W_{\alpha}^{\mu}$ and $V_{\beta}^{\nu}$, one obtains $J_{\mu \nu} \operatorname{Tr}\left[W_{\alpha}^{\mu} V_{\beta}^{\nu} \gamma_{5}\right]=0$, which means $M_{L R}=M_{R L}$. Then, the terms are given by

$$
\begin{aligned}
M_{L R}= & M_{R L} \\
= & \left(-256 J_{\mu \nu}\right)\left[\frac{M_{L R 1}^{\mu \nu}}{\left(q^{2}+2 p \cdot q\right)^{2}}+\frac{M_{L R 2}^{\mu \nu}}{\left(q^{2}+2 \bar{p} \cdot q\right)^{2}}\right. \\
& \left.+\frac{M_{L R 3}^{\mu \nu}}{\left(q^{2}+2 p \cdot q\right)\left(q^{2}+2 \bar{p} \cdot q\right)}\right], \\
M_{L R 1}^{\mu \nu}= & \frac{m_{\ell}^{2}}{2} k \cdot k^{\prime}\left\{q^{2} g^{\mu \nu}+2\left[p^{\mu} q^{\nu}+p^{\nu} q^{\mu}\right]+4 p^{\mu} p^{\nu}\right\},
\end{aligned}
$$

$$
\begin{aligned}
& M_{L R 2}^{\mu \nu}=\left(p \leftrightarrow \bar{p} \text { exchange of } M_{L R 1}^{\mu \nu}\right), \\
M_{L R 3}^{\mu \nu}= & m_{\ell}^{2}\left[g^{\mu \nu}\left(2 k \cdot q k^{\prime} \cdot q-q^{2} k \cdot k^{\prime}\right)-2 k \cdot k^{\prime}\left(p^{\mu} \bar{p}^{\nu}+p^{\nu} \bar{p}^{\mu}\right)\right. \\
- & k \cdot k^{\prime}\left(p^{\mu} q^{\nu}+p^{\nu} q^{\mu}+\bar{p}^{\mu} q^{\nu}+\bar{p}^{\nu} q^{\mu}\right) \\
+ & q^{2}\left(k^{\mu} k^{\prime \nu}+k^{\nu} k^{\prime \mu}\right)-k^{\prime} \cdot q\left(k^{\mu} q^{\nu}+k^{\nu} q^{\mu}\right) \\
- & \left.k \cdot q\left(k^{\prime \mu} q^{\nu}+k^{\prime \nu} q^{\mu}\right)\right] .
\end{aligned}
$$

When the incident neutrino is an antineutrino, the result can be obtained by replacing $k \leftrightarrow k^{\prime}$ in the above formulas. Note that the obtained transition density is invariant under the simultaneous replacement of $k \leftrightarrow k^{\prime}$ and $p \leftrightarrow \bar{p}$. These facts imply that the roles of emitted charged leptons are completely exchanged in the antineutrino case.

\section{APPENDIX B: PHASE SPACE INTEGRALS}

We perform the Monte Carlo method in calculating the four-body phase space integral $[36,37,41]$. To achieve enough convergence of the integration, we choose suitable integral variables to flatten the integrand. The phase space integrals for the four-body final state are

$$
\begin{aligned}
d \Pi= & \frac{d^{3} k^{\prime}}{(2 \pi)^{3} 2 E_{k^{\prime}}} \frac{d^{3} p}{(2 \pi)^{3} 2 E_{p}} \frac{d^{3} \bar{p}}{(2 \pi)^{3} 2 E_{\bar{p}}} \frac{d^{3} Q^{\prime}}{(2 \pi)^{3} 2 E_{Q^{\prime}}} \\
& \times(2 \pi)^{4} \delta^{(4)}\left(k^{\prime}+p+\bar{p}+Q^{\prime}-k-Q\right) .
\end{aligned}
$$

Although the number of the integration variables are $3 \times 4=12$, the net number is only eight because of the energy-momentum conservation.

In general, we can rewrite the phase space integral as

$$
\begin{aligned}
\int d \Pi= & \int_{\underline{x}_{0}}^{\bar{x}_{0}} d x_{0} \int_{\underline{x}_{1}}^{\bar{x}_{1}} d x_{1} \int_{\underline{x}_{2}}^{\bar{x}_{2}} d x_{2} \int_{\underline{x}_{3}}^{\bar{x}_{3}} d x_{3} \\
& \times \int_{\underline{x}_{4}}^{\bar{x}_{4}} d x_{4} \int_{\underline{x}_{5}}^{\bar{x}_{5}} d x_{5} \int_{\underline{x}_{6}}^{\bar{x}_{6}} d x_{6} \int_{\underline{x}_{7}}^{\bar{x}_{7}} d x_{7} \\
& \times X\left(x_{0}, x_{1}, x_{2}, x_{3}, x_{4}, x_{5}, x_{6}, x_{7}\right),
\end{aligned}
$$

where $X$ is an overall factor depending on the integral variables $x_{i}(i=0,1, \ldots, 7)$. The upper and lower limits of $x_{i}$ are represented by $\bar{x}_{i}$ and $\underline{x}_{i}$, respectively. Since it is difficult to find the range of arbitrary integral variables, we have to choose a useful set of integral variables. In our analysis, we use the following set of the integral variables $x_{i} \mathrm{~s}$ :

$$
x_{0}=\phi_{Q^{\prime}}^{(A)}
$$




$$
\begin{gathered}
x_{1}=\tau \equiv \int_{t}^{\infty} d u\{F(u)\}^{2}, \\
x_{2}=s_{p \bar{p} k} \equiv\left(p+\bar{p}+k^{\prime}\right)^{2}, \\
x_{3}=s_{\bar{p} k} \equiv\left(\bar{p}+k^{\prime}\right)^{2}, \\
x_{4}=u_{p} \equiv \log \left(-q^{2}-2 p \cdot q\right), \\
x_{5}=\phi_{p}^{(B)}, \\
x_{6}=u_{\bar{p}} \equiv \log \left(-q^{2}-2 \bar{p} \cdot q\right),
\end{gathered}
$$

$$
x_{7}=\phi_{\bar{p}}^{(C)} .
$$

$\phi_{Q^{\prime}}^{(A)}, \phi_{p}^{(B)}$, and $\phi_{\bar{p}}^{(C)}$ are rotation angles defined as follows: $\phi_{Q^{\prime}}^{(A)}$ is the rotation angle of $\boldsymbol{Q}^{\prime}$ around $\boldsymbol{k}$ in the center-ofmass frame of $\boldsymbol{k}$ and $\boldsymbol{Q}$, which we call frame $A . \phi_{p}^{(B)}$ is the rotation angle of $\boldsymbol{p}$ around $\boldsymbol{q}$ in the frame where $\boldsymbol{p}+\overline{\boldsymbol{p}}+\boldsymbol{k}^{\prime}=0$, which we call frame $B . \phi_{\bar{p}}^{(C)}$ is the rotation angle of $\overline{\boldsymbol{p}}$ around $\boldsymbol{q}$ in the frame where $\overline{\boldsymbol{p}}+\boldsymbol{k}^{\prime}=0$, which we call frame $C$. This choice of variables is useful because all of the three angles trivially run from 0 to $2 \pi$. Here, we obtain the overall factor,

$$
X=\frac{\exp \left(u_{p}+u_{\bar{p}}\right)}{(4 \pi)^{8}\left(s-M^{2}\right) \sqrt{s_{p \bar{p} k^{\prime}} s_{\bar{p} k^{\prime}}\left(t+\left|q_{0}^{(B)}\right|^{2}\right)\left(t+\left|q_{0}^{(C)}\right|^{2}\right)}\{F(t)\}^{2}} .
$$

For the NTP processes, the differential cross sections have the rotational symmetry around the neutrino beam axis. Then, the integral of $\int d \phi_{Q^{\prime}}^{(A)}$ can be simply replaced by $2 \pi$, and practically the other seven integral variables are relevant.

The variable $\tau$, defined by Eq. (B4), runs over

$$
\int_{t_{\max }}^{\infty} d u\{F(u)\}^{2}<\tau<\int_{t_{\min }}^{\infty} d u\{F(u)\}^{2}
$$

where $t_{\max }\left(t_{\min }\right)$ is the maximum (minimum) value of $t=-q^{2} \cdot t_{\max }$ and $t_{\min }$ are given by

$$
\begin{gathered}
t_{\max }=\frac{s}{2}\left(1-\frac{M^{2}}{s}\right)\left\{1-\frac{M^{2}}{s}+\sqrt{\lambda\left(1, \frac{M^{2}}{s}, \frac{4 m_{\ell}^{2}}{s}\right)}\right\} \\
-2 m_{\ell}^{2}\left(1+\frac{M^{2}}{s}\right) \\
t_{\min }=\frac{4 m_{\ell}^{2}}{t_{\max }} \frac{M^{2}}{s}
\end{gathered}
$$

Since we do not have the analytic representation of $t$ as a function of $\tau$, we prepare the numerical correspondence table between $t$ and $\tau$ for the phase space integration.

The ranges of the rest variables are as follows:

$$
\begin{gathered}
4 m_{\ell}^{2}<s_{p \bar{p} k^{\prime}}<\frac{\left(s-M^{2}\right) \sqrt{t\left(t-4 M^{2}\right)}-\left(s+M^{2}\right) t}{2 M^{2}}, \\
m_{\ell}^{2}<s_{\bar{p} k^{\prime}}<\left(\sqrt{s_{p \bar{p} k^{\prime}}}-m_{\ell}\right)^{2},
\end{gathered}
$$

$$
\begin{aligned}
\underline{u}_{p}= & \log \left(t-\frac{s_{p \bar{p} k^{\prime}}-s_{\bar{p} k^{\prime}}+m_{\ell}^{2}}{\sqrt{s_{p \bar{p} k^{\prime}}}} q_{0}^{(B)}\right. \\
& \left.-\sqrt{s_{p \bar{p} k^{\prime}} \lambda\left(1, \frac{m_{\ell}^{2}}{s_{p \bar{p} k^{\prime}}}, \frac{s_{\bar{p} k^{\prime}}}{s_{p \bar{p} k^{\prime}}}\right)\left(t+\left|q_{0}^{(B)}\right|^{2}\right)}\right), \quad(\mathrm{B} 17) \\
\bar{u}_{p}= & \log \left(t-\frac{s_{p \bar{p} k^{\prime}}-s_{\bar{p} k^{\prime}}+m_{\ell}^{2}}{\sqrt{s_{p \bar{p} k^{\prime}}}} q_{0}^{(B)}\right. \\
& \left.+\sqrt{s_{p \bar{p} k^{\prime}} \lambda\left(1, \frac{m_{\ell}^{2}}{s_{p \bar{p} k^{\prime}}}, \frac{s_{\bar{p} k^{\prime}}}{s_{p \bar{p} k^{\prime}}}\right)\left(t+\left|q_{0}^{(B)}\right|^{2}\right)}\right), \quad(\mathrm{B} 18) \\
\underline{u}_{\bar{p}}= & \log \left(t-\frac{s_{\bar{p} k^{\prime}}+m_{\ell}^{2}}{\sqrt{s_{\bar{p} k^{\prime}}}} q_{0}^{(C)}-\frac{s_{\bar{p} k^{\prime}}-m_{\ell}^{2}}{\sqrt{s_{\bar{p} k^{\prime}}}} \sqrt{t+\left|q_{0}^{(C)}\right|^{2}}\right), \\
\bar{u}_{\bar{p}}= & \log \left(t-\frac{s_{\bar{p} k^{\prime}}+m_{\ell}^{2}}{\sqrt{s_{\bar{p} k^{\prime}}}} q_{0}^{(C)}+\frac{s_{\bar{p} k^{\prime}}-m_{\ell}^{2}}{\sqrt{s_{\bar{p} k^{\prime}}}} \sqrt{t+\left|q_{0}^{(C)}\right|^{2}}\right),
\end{aligned}
$$

where $q_{0}^{(B)}$ and $q_{0}^{(C)}$ are the time components of $q$ in frames $B$ and $C$, respectively. In principle, we can derive the analytic formulas for $q_{0}^{(B)}$ and $q_{0}^{(C)}$, which are a little complicated. However, we do not need the analytic formulas because we easily obtain the numerical values of $q_{0}^{(B)}$ and $q_{0}^{(C)}$ using the step-by-step Monte Carlo integration. At each step, the upper and lower limits of $u_{p}$ are determined after $\tau, s_{p \bar{p} k^{\prime}}$, and $s_{\bar{p} k^{\prime}}$ are fixed. Then, the upper and lower limits of $u_{\bar{p}}$ are determined after $u_{p}$ and $\phi_{p}^{(B)}$ are fixed. 
[1] G. W. Bennett et al. (Muon g-2 Collaboration), Phys. Rev. D 73, 072003 (2006).

[2] M. Tanabashi et al. (Particle Data Group), Phys. Rev. D 98, 030001 (2018).

[3] T. Blum, P. A. Boyle, V. Gülpers, T. Izubuchi, L. Jin, C. Jung, A. Jüttner, C. Lehner, A. Portelli, and J. T. Tsang (RBC, UKQCD Collaborations), Phys. Rev. Lett. 121, 022003 (2018).

[4] A. Keshavarzi, D. Nomura, and T. Teubner, Phys. Rev. D 97, 114025 (2018).

[5] M. Davier, A. Hoecker, B. Malaescu, and Z. Zhang, Eur. Phys. J. C 80, 241 (2020); 80, 410(E) (2020).

[6] T. Aoyama et al., Phys. Rep. 887, 1 (2020).

[7] M. Lindner, M. Platscher, and F. S. Queiroz, Phys. Rep. 731, 1 (2018).

[8] J. Grange et al. (Muon g-2 Collaboration), arXiv: 1501.06858.

[9] M. Abe et al., Prog. Theor. Exp. Phys. 053 C02 (2019).

[10] R. Foot, Mod. Phys. Lett. A 06, 527 (1991).

[11] X.-G. He, G. C. Joshi, H. Lew, and R. R. Volkas, Phys. Rev. D 44, 2118 (1991).

[12] R. Foot, X. G. He, H. Lew, and R. R. Volkas, Phys. Rev. D 50, 4571 (1994).

[13] W. Altmannshofer, S. Gori, M. Pospelov, and I. Yavin, Phys. Rev. Lett. 113, 091801 (2014).

[14] S. Gninenko, N. Krasnikov, and V. Matveev, Phys. Rev. D 91, 095015 (2015).

[15] Y. Kaneta and T. Shimomura, Prog. Theor. Exp. Phys. 053B04 (2017).

[16] T. Araki, S. Hoshino, T. Ota, J. Sato, and T. Shimomura, Phys. Rev. D 95, 055006 (2017).

[17] C.-H. Chen and T. Nomura, Phys. Rev. D 96, 095023 (2017).

[18] T. Nomura and T. Shimomura, Eur. Phys. J. C 79, 594 (2019).

[19] H. Banerjee and S. Roy, Phys. Rev. D 99, 035035 (2019).

[20] Y. Jho, Y. Kwon, S. C. Park, and P.-Y. Tseng, J. High Energy Phys. 10 (2019) 168.

[21] S. Iguro, Y. Omura, and M. Takeuchi, J. High Energy Phys. 09 (2020) 144.

[22] d. Amaral, Dorian Warren Praia, D. G. Cerdeno, P. Foldenauer, and E. Reid, J. High Energy Phys. 12 (2020) 155.

[23] T. Araki, F. Kaneko, Y. Konishi, T. Ota, J. Sato, and T. Shimomura, Phys. Rev. D 91, 037301 (2015).

[24] T. Araki, F. Kaneko, T. Ota, J. Sato, and T. Shimomura, Phys. Rev. D 93, 013014 (2016).

[25] K. Asai, K. Hamaguchi, and N. Nagata, Eur. Phys. J. C 77, 763 (2017).

[26] K. Asai, K. Hamaguchi, N. Nagata, S.-Y. Tseng, and K. Tsumura, Phys. Rev. D 99, 055029 (2019).

[27] K. Asai, Eur. Phys. J. C 80, 76 (2020).

[28] T. Araki, K. Asai, J. Sato, and T. Shimomura, Phys. Rev. D 100, 095012 (2019).

[29] A. Kamada, K. Kaneta, K. Yanagi, and H.-B. Yu, J. High Energy Phys. 06 (2018) 117.

[30] S. Gninenko and N. Krasnikov, Phys. Lett. B 783, 24 (2018).

[31] P. Foldenauer, Phys. Rev. D 99, 035007 (2019).
[32] K. Asai, K. Hamaguchi, N. Nagata, and S.-Y. Tseng, J. Cosmol. Astropart. Phys. 11 (2020) 013.

[33] M. Ibe, W. Nakano, and M. Suzuki, Phys. Rev. D 95, 055022 (2017).

[34] Z.-L. Han, R. Ding, S.-J. Lin, and B. Zhu, Eur. Phys. J. C 79, 1007 (2019).

[35] Y. Jho, S. M. Lee, S. C. Park, Y. Park, and P.-Y. Tseng, J. High Energy Phys. 04 (2020) 086.

[36] W. Czyz, G. Sheppey, and J. Walecka, Nuovo Cimento 34, 404 (1964).

[37] J. Lovseth and M. Radomiski, Phys. Rev. D 3, 2686 (1971).

[38] K. Fujikawa, Ann. Phys. (N.Y.) 68, 102 (1971).

[39] K. Koike, M. Konuma, K. Kurata, and K. Sugano, Prog. Theor. Phys. 46, 1150 (1971).

[40] K. Koike, M. Konuma, K. Kurata, and K. Sugano, Prog. Theor. Phys. 46, 1799 (1971).

[41] R. Brown, R. Hobbs, J. Smith, and N. Stanko, Phys. Rev. D 6, 3273 (1972).

[42] R. Belusevic and J. Smith, Phys. Rev. D 37, 2419 (1988).

[43] I. Gaidaenko, V. Novikov, and M. Vysotsky, Phys. Lett. B 497, 49 (2001).

[44] W. Altmannshofer, S. Gori, M. Pospelov, and I. Yavin, Phys. Rev. D 89, 095033 (2014).

[45] D. Geiregat et al. (CHARM-II Collaboration), Phys. Lett. B 245, 271 (1990).

[46] S. R. Mishra et al. (CCFR Collaboration), Phys. Rev. Lett. 66, 3117 (1991).

[47] T. Adams et al. (NuTeV Collaboration), Phys. Rev. D 61, 092001 (2000).

[48] G. Magill and R. Plestid, Phys. Rev. D 95, 073004 (2017).

[49] G. Magill and R. Plestid, Phys. Rev. D 97, 055003 (2018).

[50] A. Falkowski, G. Grilli di Cortona, and Z. Tabrizi, J. High Energy Phys. 04 (2018) 101.

[51] P. Ballett, M. Hostert, S. Pascoli, Y. F. Perez-Gonzalez, Z. Tabrizi, and R. Zukanovich Funchal, J. High Energy Phys. 01 (2019) 119.

[52] W. Altmannshofer, S. Gori, J. Martín-Albo, A. Sousa, and M. Wallbank, Phys. Rev. D 100, 115029 (2019).

[53] P. Ballett, M. Hostert, S. Pascoli, Y. F. Perez-Gonzalez, Z. Tabrizi, and R. Zukanovich Funchal, Phys. Rev. D 100, 055012 (2019).

[54] A. de Gouvêa, P. J. Fox, R. Harnik, K. J. Kelly, and Y. Zhang, J. High Energy Phys. 01 (2019) 001.

[55] S.-F. Ge, M. Lindner, and W. Rodejohann, Phys. Lett. B 772, 164 (2017).

[56] B. Zhou and J.F. Beacom, Phys. Rev. D 101, 036010 (2020).

[57] B. Zhou and J.F. Beacom, Phys. Rev. D 101, 036011 (2020).

[58] C. De Jager, H. De Vries, and C. De Vries, At. Data Nucl. Data Tables 14, 479 (1974); 16, 580(E) (1975).

[59] R. Harnik, J. Kopp, and P. A. Machado, J. Cosmol. Astropart. Phys. 07 (2012) 026.

[60] S. K. Agarwalla, F. Lombardi, and T. Takeuchi, J. High Energy Phys. 12 (2012) 079.

[61] S. Bilmis, I. Turan, T. Aliev, M. Deniz, L. Singh, and H. Wong, Phys. Rev. D 92, 033009 (2015).

[62] J. Lees et al. (BABAR Collaboration), Phys. Rev. D 94, 011102 (2016). 